\title{
STRUCTURAL RESULTS FOR FREE ARAKI-WOODS FACTORS AND THEIR CONTINUOUS CORES
}

\author{
CYRIL HOUDAYER
}

\begin{abstract}
We show that for any type $\mathrm{III}_{1}$ free Araki-Woods factor $\mathcal{M}=\Gamma\left(H_{\mathbf{R}}, U_{t}\right)^{\prime \prime}$ associated with an orthogonal representation $\left(U_{t}\right)$ of $\mathbf{R}$ on a separable real Hilbert space $H_{\mathbf{R}}$, the continuous core $M=\mathcal{M} \rtimes_{\sigma} \mathbf{R}$ is a semisolid $\mathrm{II}_{\infty}$ factor, i.e. for any non-zero finite projection $q \in M$, the $\mathrm{II}_{1}$ factor $q M q$ is semisolid. If the representation $\left(U_{t}\right)$ is moreover assumed to be mixing, then we prove that the core $M$ is solid. As an application, we construct an example of a non-amenable solid $\mathrm{II}_{1}$ factor $N$ with full fundamental group, i.e. $\mathcal{F}(N)=\mathbf{R}_{+}^{*}$, which is not isomorphic to any interpolated free group factor $L\left(\mathbf{F}_{t}\right)$, for $1<t \leq+\infty$.
\end{abstract}

\section{Introduction and statement of the main Results}

The free Araki-Woods factors were introduced by Shlyakhtenko in 32. In the context of free probability theory, these factors can be regarded as the analogs of the hyperfinite factors coming from the CAR functor. To each real separable Hilbert space $H_{\mathbf{R}}$ together with an orthogonal representation $\left(U_{t}\right)$ of $\mathbf{R}$ on $H_{\mathbf{R}}$, one can associate a von Neumann algebra denoted by $\Gamma\left(H_{\mathbf{R}}, U_{t}\right)^{\prime \prime}$, called the free Araki-Woods von Neumann algebra. The von Neumann algebra $\Gamma\left(H_{\mathbf{R}}, U_{t}\right)^{\prime \prime}$ comes equipped with a unique free quasi-free state denoted by $\varphi_{U}$, which is always normal and faithful on $\Gamma\left(H_{\mathbf{R}}, U_{t}\right)^{\prime \prime}$ (see Section 2 for a more detailed construction). If $\operatorname{dim} H_{\mathbf{R}}=1$, then $\Gamma(\mathbf{R}, \mathrm{Id})^{\prime \prime} \cong L^{\infty}[0,1]$. If $\operatorname{dim} H_{\mathbf{R}} \geq 2$, then $\mathcal{M}=\Gamma\left(H_{\mathbf{R}}, U_{t}\right)^{\prime \prime}$ is a full factor. In particular, $\mathcal{M}$ can never be of type $\mathrm{III}_{0}$. The type classification of these factors is the following:

(1) $\mathcal{M}$ is a type $\mathrm{II}_{1}$ factor iff the representation $\left(U_{t}\right)$ is trivial: in that case the functor $\Gamma$ is Voiculescu's free Gaussian functor [38]. Then $\Gamma\left(H_{\mathbf{R}}, \mathrm{Id}\right)^{\prime \prime} \cong L\left(\mathbf{F}_{\operatorname{dim} H_{\mathbf{R}}}\right)$.

(2) $\mathcal{M}$ is a type $\operatorname{III}_{\lambda}$ factor, for $0<\lambda<1$, iff the representation $\left(U_{t}\right)$ is $\frac{2 \pi}{|\log \lambda|}$-periodic.

(3) $\mathcal{M}$ is a type $\mathrm{III}_{1}$ factor iff $\left(U_{t}\right)$ is non-periodic and non-trivial.

Using free probability techniques, Shlyakhtenko obtained several remarkable classification results for $\Gamma\left(H_{\mathbf{R}}, U_{t}\right)^{\prime \prime}$. For instance, if the orthogonal representations $\left(U_{t}\right)$ are almost periodic, then the free Araki-Woods factors $\mathcal{M}=\Gamma\left(H_{\mathbf{R}}, U_{t}\right)^{\prime \prime}$ are completely classified up to statepreserving *-isomorphism [32]: they only depend on Connes' invariant $\operatorname{Sd}(\mathcal{M})$ which is equal in that case to the (countable) subgroup $S_{U} \subset \mathbf{R}_{+}^{*}$ generated by the eigenvalues of $\left(U_{t}\right)$. Moreover, the discrete core $\mathcal{M} \rtimes_{\sigma} \widehat{S_{U}}$ (where $\widehat{S_{U}}$ is the compact group dual of $S_{U}$ ) is *isomorphic to $L\left(\mathbf{F}_{\infty}\right) \bar{\otimes} \mathbf{B}\left(\ell^{2}\right)$. Shlyakhtenko showed in 31] that if $\left(U_{t}\right)$ is the left regular representation, then the continuous core $M=\mathcal{M} \rtimes_{\sigma} \mathbf{R}$ is isomorphic to $L\left(\mathbf{F}_{\infty}\right) \bar{\otimes} \mathbf{B}\left(\ell^{2}\right)$ and the dual "trace-scaling" action $\left(\theta_{s}\right)$ is precisely the one constructed by Rădulescu [26]. For more on free Araki-Woods factors, we refer to [11, 13, 27, 28, 29, 30, 31, 32] and also to Vaes' Bourbaki seminar [37].

2000 Mathematics Subject Classification. 46L10; 46L54.

Key words and phrases. Free Araki-Woods factors; (Semi)solid factors; Deformation/Rigidity techniques; Intertwining techniques; Spectral gap property.

${ }^{1}$ Canonical Anticommutation Relations 
The free Araki-Woods factors as well as their continuous cores carry a malleable deformation in the sense of Popa. Then we will use the deformation/rigidity strategy together with the intertwining techniques in order to study the associated continuous cores. The high flexibility of this approach will allow us to work in a semifinite setting, so that we can obtain new structural/indecomposability results for the continuous cores of the free Araki-Woods factors. We first need to recall a few concepts. Following Ozawa [15, 16], a finite von Neumann algebra $N$ is said to be:

- solid if for any diffuse von Neumann subalgebra $A \subset N$, the relative commutant $A^{\prime} \cap N$ is amenable;

- semisolid if for any type $\mathrm{II}_{1}$ von Neumann subalgebra $A \subset N$, the relative commutant $A^{\prime} \cap N$ is amenable.

It is easy to check that solidity and semisolidity for $\mathrm{II}_{1}$ factors are stable under taking amplification by any $t>0$. Moreover, if $N$ is a non-amenable $\mathrm{II}_{1}$ factor, then solid $\Longrightarrow$ semisolid $\Longrightarrow$ prime. Recall in this respect that $N$ is said to be prime if it cannot be written as the tensor product of two diffuse factors.

Ozawa discovered a class $\mathcal{S}$ of countable groups for which whenever $\Gamma \in \mathcal{S}$, the group von Neumann algebra $L(\Gamma)$ is solid [15]. He showed that the following countable groups belong to the class $\mathcal{S}$ : the word-hyperbolic groups [15], the wreath products $\Lambda$ $\ulcorner$ for $\Lambda$ amenable and $\Gamma \in \mathcal{S}$ [16], and $\mathbf{Z}^{2} \rtimes \mathrm{SL}(2, \mathbf{Z})$ [17]. He moreover proved that if $\Gamma \in \mathcal{S}$, then for any free, ergodic, p.m.p. action $\Gamma \curvearrowright(X, \mu)$, the corresponding $\mathrm{II}_{1}$ factor $L^{\infty}(X, \mu) \rtimes \Gamma$ is semisolid [16]. Recall that a non-amenable solid $\mathrm{II}_{1}$ factor does not have property $\Gamma$ of Murray \& von Neumann [15].

Definition 1.1. Let $M$ be a $\mathrm{II}_{\infty}$ factor and let $\mathrm{Tr}$ be a fixed faithful normal semifinite trace on $M$. We shall say that $M$ is solid (resp. semisolid) if for any non-zero projection $q \in M$ such that $\operatorname{Tr}(q)<\infty$, the $\mathrm{II}_{1}$ factor $q M q$ is solid (resp. semisolid).

Recall that an orthogonal/unitary representation $\left(U_{t}\right)$ acting on $H$ is said to be mixing if for any $\xi, \eta \in H,\left\langle U_{t} \xi, \eta\right\rangle \rightarrow 0$, as $|t| \rightarrow \infty$. The main result of this paper is the following:

Theorem 1.2. Let $\mathcal{M}=\Gamma\left(H_{\mathbf{R}}, U_{t}\right)^{\prime \prime}$ be a type $\mathrm{III}_{1}$ free Araki-Woods factor. Then the continuous core $M=\mathcal{M} \rtimes_{\sigma} \mathbf{R}$ is a semisolid $\mathrm{II}_{\infty}$ factor. Since $M$ is non-amenable, $M$ is always a prime factor. If the representation $\left(U_{t}\right)$ is moreover assumed to be mixing, then $M$ is a solid $\mathrm{II}_{\infty}$ factor.

The proof of Theorem 1.2 follows Popa's deformation/rigidity strategy. This theory has been successfully used over the last eight years to give a plethora of new classification/rigidity results for crossed products/free products von Neumann algebras. We refer to [5, 12, 14, 19, 20, 21, 22, 23, 24, 36] for some applications of the deformation/rigidity technique. We point out that in the present paper, the rigidity part does not rely on the notion of (relative) property (T) but rather on a certain spectral gap property discovered by Popa in [19, 20]. Using this powerful technique, Popa was able to show for instance that the Bernoulli action of groups of the form $\Gamma_{1} \times \Gamma_{2}$, with $\Gamma_{1}$ non-amenable and $\Gamma_{2}$ infinite is $\mathcal{U}_{\text {fin }}$-cocycle superrigid [19]. The spectral gap rigidity principle gave also a new approach to proving primeness and (semi)solidity for type $\mathrm{II}_{1} / \mathrm{III}$ factors $4,45,19,20$. We briefly remind below the concepts that we will play against each other in order to prove Theorem 1.2 .

(1) The first ingredient we will use is the "malleable deformation" by automorphisms $\left(\alpha_{t}, \beta\right)$ defined on the free Araki-Woods factor $\mathcal{M} * \mathcal{M}=\Gamma\left(H_{\mathbf{R}} \oplus H_{\mathbf{R}}, U_{t} \oplus U_{t}\right)^{\prime \prime}$. This deformation naturally arises as the "second quantization" of the rotations/reflection defined on $H_{\mathbf{R}} \oplus H_{\mathbf{R}}$ that commute with $U_{t} \oplus U_{t}$. It was shown in 19] that such a deformation automatically features a certain "transversality property" (see Lemma 2.1 in [19]) which will be of essential use in our proof. 
(2) The second ingredient we will use is the spectral gap rigidity principle discovered by Popa in [19, 20]. Let $B \subset M_{i}$ be an inclusion of finite von Neumann algebras, for $i=1,2$, with $B$ amenable. Write $M=M_{1} *_{B} M_{2}$. Then for any von Neumann subalgebra $Q \subset M_{1}$ with no amenable direct summand, the action by conjugation $\operatorname{Ad}(\mathcal{U}(Q)) \curvearrowright M$ has "spectral gap" relative to $M_{1}$ : for any $\varepsilon>0$, there exist $\delta>0$ and a finite "critical" subset $F \subset \mathcal{U}(Q)$ such that for any $x \in(M)_{1}$ (the unit ball of $M)$, if $\left\|u x u^{*}-x\right\|_{2} \leq \delta, \forall u \in F$, then $\left\|x-E_{M_{1}}(x)\right\|_{2} \leq \varepsilon$.

(3) Let $M=\mathcal{M} \rtimes_{\sigma} \mathbf{R}$ be the continuous core the free Araki-Woods factor $\mathcal{M}$. Let $q \in M$ be a non-zero finite projection. A combination of (1) and (2) yields that for any $Q \subset q M q$ with no amenable direct summand, the malleable deformation $\left(\alpha_{t}\right)$ necessarily converges uniformly in $\|\cdot\|_{2}$ on $\left(Q^{\prime} \cap q M q\right)_{1}$. Then, using Popa's intertwining techniques, one can locate the position of $Q^{\prime} \cap q M q$ inside $q M q$.

The second result of this paper provides a new example of a non-amenable solid $\mathrm{II}_{1}$ factor. We first need the following:

Example 1.3. Using results of [2], we construct an example of an orthogonal representation $\left(U_{t}\right)$ of $\mathbf{R}$ on a (separable) real Hilbert space $K_{\mathbf{R}}$ such that:

(1) $\left(U_{t}\right)$ is mixing.

(2) The spectral measure of $\bigoplus_{n \geq 1} U_{t}^{\otimes n}$ is singular w.r.t. the Lebesgue measure on $\mathbf{R}$.

Shlyakhtenko showed in [31] that if the spectral measure of the representation $\bigoplus_{n>1} U_{t}^{\otimes n}$ is singular w.r.t. the Lebesgue measure, then the continuous core of the free Araki-Woods factor $\Gamma\left(H_{\mathbf{R}}, U_{t}\right)^{\prime \prime}$ cannot be isomorphic to any $L\left(\mathbf{F}_{t}\right) \bar{\otimes} \mathbf{B}\left(\ell^{2}\right)$, for $1<t \leq \infty$, where $L\left(\mathbf{F}_{t}\right)$ denote the interpolated free group factors [8, 25]. Therefore, we obtain:

Theorem 1.4. Let $\left(U_{t}\right)$ be an orthogonal representation acting on $K_{\mathbf{R}}$ as in Example 1.3. Denote by $\mathcal{M}=\Gamma\left(K_{\mathbf{R}}, U_{t}\right)^{\prime \prime}$ the corresponding free Araki-Woods factor and by $M=\mathcal{M} \rtimes_{\sigma} \mathbf{R}$ its continuous core. Let $q \in L(\mathbf{R})$ be a non-zero projection such that $\operatorname{Tr}(q)<\infty$. Then the non-amenable $\mathrm{II}_{1}$ factor $q M q$ is solid, has full fundamental group, i.e. $\mathcal{F}(q M q)=\mathbf{R}_{+}^{*}$, and is not isomorphic to any interpolated free group factor $L\left(\mathbf{F}_{t}\right)$, for $1<t \leq \infty$.

The paper is organized as follows. In Section 2, we recall the necessary background on free Araki-Woods factors as well as intertwining techniques for (semi)finite von Neumann algebras. Section 3 is mainly devoted to the proof of Theorem [1.2, following the deformation/spectral gap rigidity strategy presented above. In the last Section, we construct Example 1.3 and deduce Theorem 1.4.

Acknowledgement. The author would like to thank Prof. D. Shlyakhtenko for suggesting him the idea of looking at indecomposability results for the continuous cores of the free ArakiWoods factors and also for the useful discussions.

\section{Preliminary BACKGRound}

2.1. Shlyakhtenko's free Araki-Woods factors. Let $H_{\mathbf{R}}$ be a real separable Hilbert space and let $\left(U_{t}\right)$ be an orthogonal representation of $\mathbf{R}$ on $H_{\mathbf{R}}$ such that the map $t \mapsto U_{t}$ is strongly continuous. Let $H_{\mathbf{C}}=H_{\mathbf{R}} \otimes_{\mathbf{R}} \mathbf{C}$ be the complexified Hilbert space. We shall still denote by $\left(U_{t}\right)$ the corresponding unitary representation of $\mathbf{R}$ on $H_{\mathbf{C}}$. Let $A$ be the infinitesimal generator of $\left(U_{t}\right)$ on $H_{\mathbf{C}}$ (Stone's theorem), so that $A$ is the positive, self-adjoint, (possibly) unbounded operator on $H_{\mathbf{C}}$ which satisfies $U_{t}=A^{i t}$, for every $t \in \mathbf{R}$. Define another inner product on $H_{\mathbf{C}}$ by

$$
\langle\xi, \eta\rangle_{U}=\left\langle\frac{2}{1+A^{-1}} \xi, \eta\right\rangle, \forall \xi, \eta \in H_{\mathbf{C}}
$$

Note that for any $\xi \in H_{\mathbf{R}},\|\xi\|_{U}=\|\xi\|$; also, for any $\xi, \eta \in H_{\mathbf{R}}, \Re\left(\langle\xi, \eta\rangle_{U}\right)=\langle\xi, \eta\rangle$, where $\Re$ denotes the real part. Denote by $H$ the completion of $H_{\mathbf{C}}$ w.r.t. the new inner product 
$\langle\cdot, \cdot\rangle_{U}$, and note that $\left(U_{t}\right)$ is still a unitary representation on $H$. Introduce now the full Fock space of $H$ :

$$
\mathcal{F}(H)=\mathbf{C} \Omega \oplus \bigoplus_{n=1}^{\infty} H^{\bar{\otimes} n}
$$

The unit vector $\Omega$ is called the vacuum vector. For any $\xi \in H$, we have the left creation operator

$$
\ell(\xi): \mathcal{F}(H) \rightarrow \mathcal{F}(H):\left\{\begin{array}{l}
\ell(\xi) \Omega=\xi \\
\ell(\xi)\left(\xi_{1} \otimes \cdots \otimes \xi_{n}\right)=\xi \otimes \xi_{1} \otimes \cdots \otimes \xi_{n} .
\end{array}\right.
$$

For any $\xi \in H$, we denote by $s(\xi)$ the real part of $\ell(\xi)$ given by

$$
s(\xi)=\frac{\ell(\xi)+\ell(\xi)^{*}}{2} .
$$

The crucial result of Voiculescu [38] is that the distribution of the operator $s(\xi)$ w.r.t. the vacuum vector state $\varphi_{U}=\langle\cdot \Omega, \Omega\rangle_{U}$ is the semicircular law of Wigner supported on the interval $[-\|\xi\|,\|\xi\|]$.

Definition 2.1 (Shlyakhtenko, [32]). Let $\left(U_{t}\right)$ be an orthogonal representation of $\mathbf{R}$ on the real Hilbert space $H_{\mathbf{R}}$. The free Araki-Woods von Neumann algebra associated with $H_{\mathbf{R}}$ and $\left(U_{t}\right)$, denoted by $\Gamma\left(H_{\mathbf{R}}, U_{t}\right)^{\prime \prime}$, is defined by

$$
\Gamma\left(H_{\mathbf{R}}, U_{t}\right)^{\prime \prime}:=\left\{s(\xi): \xi \in H_{\mathbf{R}}\right\}^{\prime \prime} .
$$

The vector state $\varphi_{U}=\langle\cdot \Omega, \Omega\rangle_{U}$ is called the free quasi-free state. It is normal and faithful on $\Gamma\left(H_{\mathbf{R}}, U_{t}\right)^{\prime \prime}$.

Recall that for any type $\mathrm{III}_{1}$ factor $\mathcal{M}$, Connes-Takesaki's continuous decomposition [7, 33 yields

$$
\mathcal{M} \bar{\otimes} \mathbf{B}\left(L^{2}(\mathbf{R})\right) \cong\left(\mathcal{M} \rtimes_{\sigma} \mathbf{R}\right) \rtimes_{\theta} \mathbf{R},
$$

where the continuous core $\mathcal{M} \rtimes_{\sigma} \mathbf{R}$ is a $\mathrm{II}_{\infty}$ factor and $\theta$ is the trace-scaling action [33]:

$$
\operatorname{Tr}\left(\theta_{s}(x)\right)=e^{-s} \operatorname{Tr}(x), \forall x \in\left(\mathcal{M} \rtimes_{\sigma} \mathbf{R}\right)_{+}, \forall s \in \mathbf{R} .
$$

The fact that $\mathcal{M} \rtimes_{\sigma} \mathbf{R}$ does not depend on the choice of a f.n. state on $\mathcal{M}$ follows from Connes' Radon-Nikodym derivative theorem [7]. Moreover, for any non-zero finite projection $q \in M=\mathcal{M} \rtimes_{\sigma} \mathbf{R}$, the $\mathrm{II}_{1}$ factor $q M q$ has full fundamental group.

Following [6], a factor $\mathcal{M}$ (with separable predual) is said to be full if the subgroup of inner automorphisms $\operatorname{Inn}(\mathcal{M}) \subset \operatorname{Aut}(\mathcal{M})$ is closed. Recall that $\operatorname{Aut}(\mathcal{M})$ is endowed with the $u$-topology: for any sequence $\left(\theta_{n}\right)$ in $\operatorname{Aut}(\mathcal{M})$,

$$
\theta_{n} \rightarrow \mathrm{Id}, \text { as } n \rightarrow \infty \Longleftrightarrow\left\|\varphi \circ \theta_{n}-\varphi\right\| \rightarrow 0 \text {, as } n \rightarrow \infty, \forall \varphi \in \mathcal{M}_{*} .
$$

Since $\mathcal{M}$ has a separable predual, $\operatorname{Aut}(\mathcal{M})$ is a polish group. For any $\mathrm{II}_{1}$ factor $N, N$ is full iff $N$ does not have property $\Gamma$ of Murray \& von Neumann (see [6]).

Denote by $\pi: \operatorname{Aut}(\mathcal{M}) \rightarrow \operatorname{Out}(\mathcal{M})$ the canonical projection. Assume $\mathcal{M}$ is a full factor so that $\operatorname{Out}(\mathcal{M})$ is a Hausdorff topological group. Fix a f.n. state $\varphi$ on $\mathcal{M}$. Connes' invariant $\tau(\mathcal{M})$ is defined as the weakest topology on $\mathbf{R}$ that makes the map

$$
\begin{aligned}
\mathbf{R} & \rightarrow \operatorname{Out}(\mathcal{M}) \\
t & \mapsto \pi\left(\sigma_{t}^{\varphi}\right)
\end{aligned}
$$

continuous. Note that this map does not depend on the choice of the f.n. state $\varphi$ on $\mathcal{M}$ [7.

Denote by $\mathcal{F}\left(U_{t}\right)=\bigoplus_{n \in \mathbf{N}} U_{t}^{\otimes n}$. The modular group $\sigma^{\varphi_{U}}$ of the free quasi-free state is given by: $\sigma_{t}^{\varphi_{U}}=\operatorname{Ad}\left(\mathcal{F}\left(U_{-t}\right)\right)$, for any $t \in \mathbf{R}$. The free Araki-Woods factors provided many new examples of full factors of type III [3, 7, 28]. We can summarize their general properties in the following theorem (see also Vaes' Bourbaki seminar [37]): 
Theorem 2.2 (Shlyakhtenko, 28, 30, 31, 32]). Let $\left(U_{t}\right)$ be an orthogonal representation of $\mathbf{R}$ on the real Hilbert space $H_{\mathbf{R}}$ with $\operatorname{dim} H_{\mathbf{R}} \geq 2$. Denote by $\mathcal{M}=\Gamma\left(H_{\mathbf{R}}, U_{t}\right)^{\prime \prime}$.

(1) $\mathcal{M}$ is a full factor and Connes' invariant $\tau(\mathcal{M})$ is the weakest topology on $\mathbf{R}$ that makes the map $t \mapsto U_{t}$ strongly continuous.

(2) $\mathcal{M}$ is of type $\mathrm{II}_{1}$ iff $U_{t}=\mathrm{id}$ for every $t \in \mathbf{R}$. In this case, $\mathcal{M} \cong L\left(\mathbf{F}_{\operatorname{dim}\left(H_{\mathbf{R}}\right)}\right)$.

(3) $\mathcal{M}$ is of type $\operatorname{III}_{\lambda}(0<\lambda<1)$ iff $\left(U_{t}\right)$ is periodic of period $\frac{2 \pi}{|\log \lambda|}$.

(4) $\mathcal{M}$ is of type III $_{1}$ in the other cases.

(5) $\mathcal{M}$ has almost periodic states iff $\left(U_{t}\right)$ is almost periodic.

Moreover, it follows from 27 that any free Araki-Woods factor $\mathcal{M}$ is generalized solid in the sense of [35]: for any diffuse von Neumann subalgebra $A \subset \mathcal{M}$ such that there exists a faithful normal conditional expectation $E: \mathcal{M} \rightarrow A$, the relative commutant $A^{\prime} \cap \mathcal{M}$ is amenable.

Notice that the centralizer of the free quasi-free state $\mathcal{M}^{\varphi_{U}}$ may be trivial. This is the case for instance when the representation $\left(U_{t}\right)$ has no eigenvectors. Nevertheless, the author recently proved in [11] that for any type $\mathrm{III}_{1}$ free Araki-Woods factor $\mathcal{M}$, the bicentralizer is trivial, i.e. there always exists a faithful normal state $\psi$ on $\mathcal{M}$ such that $\left(\mathcal{M}^{\psi}\right)^{\prime} \cap \mathcal{M}=\mathbf{C}$. We refer to [10] for more on Connes' bicentralizer problem.

Remark 2.3 ([32]). Explicitly the value of $\varphi_{U}$ on a word in $s\left(\xi_{\iota}\right)$ is given by

$$
\varphi_{U}\left(s\left(\xi_{1}\right) \cdots s\left(\xi_{n}\right)\right)=2^{-n} \sum_{\left(\left\{\beta_{i}, \gamma_{i}\right\}\right) \in \mathrm{NC}(n), \beta_{i}<\gamma_{i}} \prod_{k=1}^{n / 2}\left\langle\xi_{\beta_{k}}, \xi_{\gamma_{k}}\right\rangle_{U} .
$$

for $n$ even and is zero otherwise. Here $\mathrm{NC}(2 p)$ stands for all the non-crossing pairings of the set $\{1, \ldots, 2 p\}$, i.e. pairings for which whenever $a<b<c<d$, and $a, c$ are in the same class, then $b, d$ are not in the same class. The total number of such pairings is given by the $p$-th Catalan number

$$
C_{p}=\frac{1}{p+1}\left(\begin{array}{c}
2 p \\
p
\end{array}\right)
$$

Recall that a continuous $\varphi$-preserving action $\left(\sigma_{t}\right)$ of $\mathbf{R}$ on a von Neumann algebra $\mathcal{M}$ endowed with a f.n. state $\varphi$ is said to be $\varphi$-mixing if for any $x, y \in \mathcal{M}$ with $\varphi(x)=\varphi(y)=0$,

$$
\varphi\left(\sigma_{t}(x) y\right) \rightarrow 0, \text { as }|t| \rightarrow \infty .
$$

Proposition 2.4. Let $\mathcal{M}=\Gamma\left(H_{\mathbf{R}}, U_{t}\right)^{\prime \prime}$ be any free Araki-Woods factor and let $\varphi_{U}$ be the free quasi-free state. Then

$$
\left(U_{t}\right) \text { is mixing } \Longleftrightarrow\left(\sigma_{t}^{\varphi_{U}}\right) \text { is } \varphi_{U} \text {-mixing. }
$$

Proof. We prove both directions.

$\Longleftarrow$ For any $\xi, \eta \in H_{\mathbf{R}}, \varphi_{U}(s(\xi))=\varphi_{U}(s(\eta))=0$. Moreover,

$$
\begin{aligned}
\left\langle U_{t} \xi, \eta\right\rangle_{U} & =4 \varphi_{U}\left(s\left(U_{t} \xi\right) s(\eta)\right) \\
& =4 \varphi_{U}\left(\sigma_{-t}^{\varphi_{U}}(s(\xi)) s(\eta)\right) \rightarrow 0, \text { as }|t| \rightarrow \infty .
\end{aligned}
$$

It follows that $\left(U_{t}\right)$ is mixing.

$\Longrightarrow$ One needs to show that for any $x, y \in \mathcal{M}$,

$$
\lim _{|t| \rightarrow \infty} \varphi_{U}\left(\sigma_{t}^{\varphi_{U}}(x) y\right)=\varphi_{U}(x) \varphi_{U}(y) .
$$

Note that

$$
\operatorname{span}\left\{1, s\left(\xi_{1}\right) \cdots s\left(\xi_{n}\right): n \geq 1, \xi_{1}, \ldots, \xi_{n} \in H_{\mathbf{R}}\right\}
$$


is a unital $*$-strongly dense $*$-subalgebra of $\mathcal{M}$. Using Kaplansky density theorem, it suffices to check Equation (2) for $x, y \in \mathcal{M}$ of the following form:

$$
\begin{aligned}
& x=s\left(\xi_{1}\right) \cdots s\left(\xi_{p}\right) \\
& y=s\left(\eta_{1}\right) \cdots s\left(\eta_{q}\right) .
\end{aligned}
$$

Assume that $p+q$ is odd. Then $p$ or $q$ is odd and we have $\varphi_{U}\left(\sigma_{t}^{\varphi_{U}}(x) y\right)=0=\varphi_{U}(x) \varphi_{U}(y)$, for any $t \in \mathbf{R}$.

Assume now that $p+q$ is even.

(1) Suppose that $p, q$ are odd and write $p=2 k+1, q=2 l+1$. Then

$$
\begin{aligned}
\varphi_{U}\left(\sigma_{t}^{\varphi_{U}}(x) y\right) & =\varphi_{U}\left(s\left(U_{-t} \xi_{1}\right) \cdots s\left(U_{-t} \xi_{2 k+1}\right) s\left(\eta_{1}\right) \cdots s\left(\eta_{2 l+1}\right)\right) \\
& =2^{-2(k+l+1)} \sum_{\left(\left\{\beta_{i}, \gamma_{i}\right\}\right) \in \mathrm{NC}(2(k+l+1)), \beta_{i}<\gamma_{i}} \prod_{j=1}^{k+l+1}\left\langle h_{\beta_{j}}, h_{\gamma_{j}}\right\rangle_{U},
\end{aligned}
$$

where the letter $h$ stands for $U_{-t} \xi$ or $\eta$. Notice that since $2 k+1$ and $2 l+1$ are odd, for any non-crossing pairing $\left(\left\{\beta_{i}, \gamma_{i}\right\}\right) \in \mathrm{NC}(2(k+l+1))$, there must exist some $j \in\{1, \ldots, k+l+1\}$ such that $\left\langle h_{\beta_{j}}, h_{\gamma_{j}}\right\rangle=\left\langle U_{-t} \xi_{\beta_{j}}, \eta_{\gamma_{j}}\right\rangle$. Since we assumed that $\left(U_{t}\right)$ is mixing, it follows that $\varphi_{U}\left(\sigma_{t}^{\varphi_{U}}(x) y\right) \rightarrow 0=\varphi_{U}(x) \varphi_{U}(y)$, as $|t| \rightarrow \infty$.

(2) Suppose that $p, q$ are even and write $p=2 k, q=2 l$. Then

$$
\begin{aligned}
\varphi_{U}\left(\sigma_{t}^{\varphi_{U}}(x) y\right) & =\varphi_{U}\left(s\left(U_{-t} \xi_{1}\right) \cdots s\left(U_{-t} \xi_{2 k}\right) s\left(\eta_{1}\right) \cdots s\left(\eta_{2 l}\right)\right) \\
& =2^{-2(k+l)} \sum_{\left(\left\{\beta_{i}, \gamma_{i}\right\}\right) \in \mathrm{NC}(2(k+l)), \beta_{i}<\gamma_{i}} \prod_{j=1}^{k+l}\left\langle h_{\beta_{j}}, h_{\gamma_{j}}\right\rangle_{U},
\end{aligned}
$$

where the letter $h$ stands for $U_{-t} \xi$ or $\eta$. Note that for a non-crossing pairing $\nu=$ $\left(\left\{\beta_{i}, \gamma_{i}\right\}\right) \in \mathrm{NC}(2(k+l))$ such that an element of $\{1, \ldots, 2 k\}$ and an element of $\{1, \ldots, 2 l\}$ are in the same class, the proof of (1) yields that the corresponding product $\prod_{j=1}^{k+l}\left\langle h_{\beta_{j}}, h_{\gamma_{j}}\right\rangle_{U}$ goes to 0 , as $|t| \rightarrow \infty$. Thus, we just need to sum up over the noncrossing pairings $\nu$ of the form $\nu_{1} \times \nu_{2}$, where $\nu_{1}$ is a non-crossing pairing on the set $\{1, \ldots, 2 k\}$ and $\nu_{2}$ is a non-crossing pairing on the set $\{1, \ldots, 2 l\}$. Consequently, we get $\varphi_{U}\left(\sigma_{t}^{\varphi_{U}}(x) y\right) \rightarrow \varphi_{U}(x) \varphi_{U}(y)$, as $|t| \rightarrow \infty$.

Therefore, $\left(\sigma_{t}^{\varphi_{U}}\right)$ is mixing.

Proposition 2.5. Let $\mathcal{M}=\Gamma\left(H_{\mathbf{R}}, U_{t}\right)^{\prime \prime}$. If $\left(U_{t}\right)$ is mixing, then Connes' invariant $\tau(\mathcal{M})$ is the usual topology on $\mathbf{R}$.

Proof. Let $\mathcal{M}=\Gamma\left(H_{\mathbf{R}}, U_{t}\right)^{\prime \prime}$. Recall from Theorem 2.2 that $\tau(\mathcal{M})$ is the weakest topology on $\mathbf{R}$ that makes the map $t \mapsto U_{t}$ strongly continuous. Let $\left(t_{k}\right)$ be a sequence in $\mathbf{R}$ such that $t_{k} \rightarrow 0$ w.r.t. the topology $\tau(\mathcal{M})$, as $k \rightarrow \infty$, i.e. $U_{t_{k}} \rightarrow$ Id strongly, as $k \rightarrow \infty$. Fix $\xi \in H_{\mathbf{R}}$, $\|\xi\|=1$. Since

$$
\lim _{k \rightarrow \infty}\left\langle U_{t_{k}} \xi, \xi\right\rangle=1
$$

and $\left(U_{t}\right)$ is assumed to be mixing, it follows that $\left(t_{k}\right)$ is necessarily bounded. Let $t \in \mathbf{R}$ be any cluster point for the sequence $\left(t_{k}\right)$. Then $U_{t}=\mathrm{Id}$. Since $\left(U_{t}\right)$ is mixing, it follows that $t=0$. Therefore $\left(t_{k}\right)$ converges to 0 w.r.t. the usual topology on $\mathbf{R}$.

2.2. Intertwining techniques for (semi)finite von Neumann algebras. Let $(B, \tau)$ be a finite von Neumann algebra with a distinguished f.n. trace. Since $\tau$ is fixed, we simply denote $L^{2}(B, \tau)$ by $L^{2}(B)$. Let $H$ be a right Hilbert $B$-module, i.e. $H$ is a complex (separable) Hilbert space together with a normal $*$-representation $\pi: B^{\text {op }} \rightarrow \mathbf{B}(H)$. For any $b \in B$, and $\xi \in H$, we shall simply write $\pi\left(b^{\text {op }}\right) \xi=\xi b$. By the general theory, we know that there exists an isometry $v: H \rightarrow \ell^{2} \bar{\otimes} L^{2}(B)$ such that $v(\xi b)=v(\xi) b$, for any $\xi \in H, b \in B$. Since $p=v v^{*}$ 
commutes with the right $B$-action on $\ell^{2} \bar{\otimes} L^{2}(B)$, it follows that $p \in \mathbf{B}\left(\ell^{2}\right) \bar{\otimes} B$. Thus, as right $B$-modules, we have $H_{B} \simeq p\left(\ell^{2} \bar{\otimes} L^{2}(B)\right)_{B}$.

On $\mathbf{B}\left(\ell^{2}\right) \bar{\otimes} B$, we define the following f.n. semifinite trace $\operatorname{Tr}$ (which depends on $\tau$ ): for any $x=\left[x_{i j}\right]_{i, j} \in\left(\mathbf{B}\left(\ell^{2}\right) \bar{\otimes} B\right)_{+}$,

$$
\operatorname{Tr}\left(\left[x_{i j}\right]_{i, j}\right)=\sum_{i} \tau\left(x_{i i}\right) .
$$

We set $\operatorname{dim}\left(H_{B}\right)=\operatorname{Tr}\left(v v^{*}\right)$. Note that the dimension of $H$ depends on $\tau$ but does not depend on the isometry $v$. Indeed take another isometry $w: H \rightarrow \ell^{2} \bar{\otimes} L^{2}(B)$, satisfying $w(\xi b)=w(\xi) b$, for any $\xi \in H, b \in B$. Note that $v w^{*} \in \mathbf{B}\left(\ell^{2}\right) \bar{\otimes} B$ and $w^{*} w=v^{*} v=1$. Thus, we have

$$
\operatorname{Tr}\left(v v^{*}\right)=\operatorname{Tr}\left(v w^{*} w v^{*}\right)=\operatorname{Tr}\left(w v^{*} v w^{*}\right)=\operatorname{Tr}\left(w w^{*}\right) .
$$

Assume that $\operatorname{dim}\left(H_{B}\right)<\infty$. Then for any $\varepsilon>0$, there exists a central projection $z \in$ $\mathcal{Z}(B)$, with $\tau(z) \geq 1-\varepsilon$, such that the right $B$-module $H z$ is finitely generated, i.e. of the form $p L^{2}(B)^{\oplus n}$ for some projection $p \in \mathbf{M}_{n}(\mathbf{C}) \otimes B$. The non-normalized trace on $\mathbf{M}_{n}(\mathbf{C})$ will be denoted by $\operatorname{Tr}_{n}$. For simplicity, we shall denote $B^{n}:=\mathbf{M}_{n}(\mathbf{C}) \otimes B$.

In 21, 22, Popa introduced a powerful tool to prove the unitary conjugacy of two von Neumann subalgebras of a tracial von Neumann algebra $(M, \tau)$. If $A, B \subset(M, \tau)$ are two (possibly non-unital) von Neumann subalgebras, denote by $1_{A}, 1_{B}$ the units of $A$ and $B$. Note that we endow the finite von Neumann algebra $B$ with the trace $\tau\left(1_{B} \cdot 1_{B}\right) / \tau\left(1_{B}\right)$.

Theorem 2.6 (Popa, [21, 22]). Let $A, B \subset(M, \tau)$ be two (possibly non-unital) embeddings. The following are equivalent:

(1) There exist $n \geq 1$, a (possibly non-unital) $*$-homomorphism $\psi: A \rightarrow B^{n}$ and a nonzero partial isometry $v \in \mathbf{M}_{1, n}(\mathbf{C}) \otimes 1_{A} M 1_{B}$ such that $x v=v \psi(x)$, for any $x \in A$.

(2) The bimodule ${ }_{A} L^{2}\left(1_{A} M 1_{B}\right)_{B}$ contains a non-zero sub-bimodule ${ }_{A} H_{B}$ which satisfies $\operatorname{dim}\left(H_{B}\right)<\infty$.

(3) There is no sequence of unitaries $\left(u_{k}\right)$ in $A$ such that $\left\|E_{B}\left(a^{*} u_{k} b\right)\right\|_{2} \rightarrow 0$, as $k \rightarrow \infty$, for any $a, b \in 1_{A} M 1_{B}$.

If one of the previous equivalent conditions is satisfied, we shall say that $A$ embeds into $B$ inside $M$ and denote $A \preceq_{M} B$.

Definition 2.7 (Popa \& Vaes, 24). Let $A \subset B \subset(N, \tau)$ be an inclusion of finite von Neumann algebras. We say that $B \subset N$ is weakly mixing through $A$ if there exists a sequence of unitaries $\left(u_{k}\right)$ in $A$ such that

$$
\left\|E_{B}\left(a^{*} u_{k} b\right)\right\|_{2} \rightarrow 0, \text { as } k \rightarrow \infty, \forall a, b \in N \ominus B .
$$

The following result will be a crucial tool in Section 3 ; it will allow us to control the relative commutant $A^{\prime} \cap N$ of certain subalgebras $A$ of a given von Neumann algebra $N$.

Theorem 2.8 (Popa, 21]). Let $(N, \tau)$ be a finite von Neumann algebra and $A \subset B \subset N$ be von Neumann subalgebras. Assume that $B \subset N$ is weakly mixing through $A$. Then for any sub-bimodule ${ }_{A} H_{B}$ of ${ }_{A} L^{2}(N)_{B}$ such that $\operatorname{dim}\left(H_{B}\right)<\infty$, one has $H \subset L^{2}(B)$. In particular, $A^{\prime} \cap N \subset B$.

For our purpose, we will need to use Popa's intertwining techniques for semifinite von Neumann algebras. We refer to Section 2 of [5] where such techniques were developed. Namely, let $(M, T r)$ be a von Neumann algebra endowed with a faithful normal semifinite trace Tr. We shall simply denote by $L^{2}(M)$ the $M-M$ bimodule $L^{2}(M, \operatorname{Tr})$, and by $\|\cdot\|_{2, \operatorname{Tr}}$ the $L^{2}$-norm associated with the trace Tr. We will use quite often the following inequality:

$$
\|x \eta y\|_{2, \operatorname{Tr}} \leq\|x\|_{\infty}\|y\|_{\infty}\|\eta\|_{2, \operatorname{Tr}}, \forall \eta \in L^{2}(M), \forall x, y \in M
$$


where $\|\cdot\|_{\infty}$ denotes the operator norm. We shall say that a projection $p \in M$ is Tr-finite if $\operatorname{Tr}(p)<\infty$. Note that a non-zero $\operatorname{Tr}$-finite projection $p$ is necessarily finite and $\operatorname{Tr}(p \cdot p) / \operatorname{Tr}(p)$ is a f.n. (finite) trace on $p M p$. Remind that for any projections $p, q \in M$, we have $p \vee q-p \sim$ $q-p \wedge q$. Then it follows that for any Tr-finite projections $p, q \in M, p \vee q$ is still Tr-finite and $\operatorname{Tr}(p \vee q)=\operatorname{Tr}(p)+\operatorname{Tr}(q)-\operatorname{Tr}(p \wedge q)$.

Note that if a sequence $\left(x_{k}\right)$ in $M$ converges to 0 strongly, as $k \rightarrow \infty$, then for any non-zero Tr-finite projection $q \in M,\left\|x_{k} q\right\|_{2, \operatorname{Tr}} \rightarrow 0$, as $k \rightarrow \infty$. Indeed,

$$
\begin{aligned}
x_{k} \rightarrow 0 \text { strongly in } M & \Longleftrightarrow x_{k}^{*} x_{k} \rightarrow 0 \text { weakly in } M \\
& \Longrightarrow q x_{k}^{*} x_{k} q \rightarrow 0 \text { weakly in } q M q \\
& \Longrightarrow \operatorname{Tr}\left(q x_{k}^{*} x_{k} q\right) \rightarrow 0 \\
& \Longrightarrow\left\|x_{k} q\right\|_{2, \mathrm{Tr}} \rightarrow 0 .
\end{aligned}
$$

Moreover, there always exists an increasing sequence of Tr-finite projections $\left(p_{k}\right)$ in $M$ such that $p_{k} \rightarrow 1$ strongly, as $k \rightarrow \infty$.

Theorem 2.9 (5]). Let $(M, \operatorname{Tr})$ be a semifinite von Neumann algebra. Let $B \subset M$ be a von Neumann subalgebra such that $\operatorname{Tr}_{\mid B}$ is still semifinite. Denote by $E_{B}: M \rightarrow B$ the unique Tr-preserving faithful normal conditional expectation. Let $q \in M$ be a non-zero Trfinite projection. Let $A \subset q M q$ be a von Neumann subalgebra. The following conditions are equivalent:

(1) There exists a Tr-finite projection $p \in B, p \neq 0$, such that the bimodule ${ }_{A} L^{2}(q M p)_{p B p}$ contains a non-zero sub-bimodule ${ }_{A} H_{p B p}$ which satisfies $\operatorname{dim}\left(H_{p B p}\right)<\infty$, where $p B p$ is endowed with the finite trace $\operatorname{Tr}(p \cdot p) / \operatorname{Tr}(p)$.

(2) There is no sequence of unitaries $\left(u_{k}\right)$ in $A$ such that $E_{B}\left(x^{*} u_{k} y\right) \rightarrow 0$ strongly, as $k \rightarrow \infty$, for any $x, y \in q M$.

Definition 2.10. Under the assumptions of Theorem 2.9, if one of the equivalent conditions is satisfied, we shall still say that $A$ embeds into $B$ inside $M$ and still denote $A \preceq_{M} B$.

\section{Structural Results for the Continuous Cores of $\Gamma\left(H_{\mathbf{R}}, U_{t}\right)^{\prime \prime}$}

3.1. Deformation/spectral gap rigidity strategy. We first introduce some notation we will be using throughout this section. Let $H_{\mathbf{R}}$ be a separable real Hilbert space $\left(\operatorname{dim}\left(H_{\mathbf{R}}\right) \geq 2\right)$ and let $\left(U_{t}\right)$ be an orthogonal representation of $\mathbf{R}$ on $H_{\mathbf{R}}$ that we assume to be neither trivial nor periodic. We set:

- $\mathcal{M}=\Gamma\left(H_{\mathbf{R}}, U_{t}\right)^{\prime \prime}$ is the free Araki-Woods factor associated with $\left(H_{\mathbf{R}}, U_{t}\right), \varphi$ is the free quasi-free state and $\sigma$ is the modular group of the state $\varphi$. $\mathcal{M}$ is necessarily a type $\mathrm{III}_{1}$ factor since $\left(U_{t}\right)$ is neither periodic nor trivial.

- $M=\mathcal{M} \rtimes_{\sigma} \mathbf{R}$ is the continuous core of $\mathcal{M}$ and $\operatorname{Tr}$ is the semifinite trace associated with the state $\varphi . M$ is a $\mathrm{II}_{\infty}$ factor since $\mathcal{M}$ is a type $\mathrm{III}_{1}$ factor.

- Likewise $\widetilde{\mathcal{M}}=\Gamma\left(H_{\mathbf{R}} \oplus H_{\mathbf{R}}, U_{t} \oplus U_{t}\right)^{\prime \prime}, \widetilde{\varphi}$ is the corresponding free quasi-free state and $\widetilde{\sigma}$ is the modular group of $\widetilde{\varphi}$.

- $\widetilde{M}=\widetilde{\mathcal{M}} \rtimes_{\widetilde{\sigma}} \mathbf{R}$ is the continuous core of $\widetilde{\mathcal{M}}$ and $\widetilde{T r}$ is the f.n. semifinite trace associated with $\widetilde{\varphi}$.

It follows from [32] that

$$
(\widetilde{\mathcal{M}}, \widetilde{\varphi}) \cong(\mathcal{M}, \varphi) *(\mathcal{M}, \varphi) .
$$

In the latter free product, we shall write $\mathcal{M}_{1}$ for the first copy of $\mathcal{M}$ and $\mathcal{M}_{2}$ for the second copy of $\mathcal{M}$. We regard $\mathcal{M} \subset \widetilde{\mathcal{M}}$ via the identification of $\mathcal{M}$ with $\mathcal{M}_{1}$.

Denote by $\left(\lambda_{t}\right)$ the unitaries in $L(\mathbf{R})$ that implement the modular action $\sigma$ on $\mathcal{M}$ (resp. $\widetilde{\sigma}$ on $\widetilde{\mathcal{M}})$. Define the following faithful normal conditional expectations: 
- $E: M \rightarrow L(\mathbf{R})$ such that $E\left(x \lambda_{t}\right)=\varphi(x) \lambda_{t}$, for every $x \in \mathcal{M}$ and $t \in \mathbf{R}$;

- $\widetilde{E}: \widetilde{M} \rightarrow L(\mathbf{R})$ such that $\widetilde{E}\left(x \lambda_{t}\right)=\widetilde{\varphi}(x) \lambda_{t}$, for every $x \in \widetilde{\mathcal{M}}$ and $t \in \mathbf{R}$.

Then

$$
(\widetilde{M}, \widetilde{E}) \cong(M, E) *_{L(\mathbf{R})}(M, E) .
$$

Likewise, in the latter amalgamated free product, we shall write $M_{1}$ for the first copy of $M$ and $M_{2}$ for the second copy of $M$. We regard $M \subset \widetilde{M}$ via the identification of $M$ with $M_{1}$. Notice that the conditional expectation $E$ (resp. $\widetilde{E}$ ) preserves the canonical semifinite trace $\operatorname{Tr}($ resp. $\widetilde{\operatorname{Tr}}$ ) associated with the state $\varphi$ (resp. $\widetilde{\varphi})$ (see [34]).

Consider the following orthogonal representation of $\mathbf{R}$ on $H_{\mathbf{R}} \oplus H_{\mathbf{R}}$ :

$$
V_{s}=\left(\begin{array}{cc}
\cos \left(\frac{\pi}{2} s\right) & -\sin \left(\frac{\pi}{2} s\right) \\
\sin \left(\frac{\pi}{2} s\right) & \cos \left(\frac{\pi}{2} s\right)
\end{array}\right), \forall s \in \mathbf{R} .
$$

Let $\left(\alpha_{s}\right)$ be the natural action on $(\widetilde{\mathcal{M}}, \widetilde{\varphi})$ associated with $\left(V_{s}\right): \alpha_{s}=\operatorname{Ad}\left(\mathcal{F}\left(V_{s}\right)\right)$, for every $s \in \mathbf{R}$. In particular, we have

$$
\alpha_{s}\left(s\left(\begin{array}{l}
\xi \\
\eta
\end{array}\right)\right)=s\left(V_{s}\left(\begin{array}{l}
\xi \\
\eta
\end{array}\right)\right), \forall s \in \mathbf{R}, \forall \xi, \eta \in H_{\mathbf{R}},
$$

and the action $\left(\alpha_{s}\right)$ is $\widetilde{\varphi}$-preserving. We can easily see that the representation $\left(V_{s}\right)$ commutes with the representation $\left(U_{t} \oplus U_{t}\right)$. Consequently, $\left(\alpha_{s}\right)$ commutes with modular action $\widetilde{\sigma}$. Moreover, $\alpha_{1}(x * 1)=1 * x$, for every $a \in \mathcal{M}$. At last, consider the automorphism $\beta$ defined on $(\widetilde{\mathcal{M}}, \widetilde{\varphi})$ by:

$$
\beta\left(s\left(\begin{array}{c}
\xi \\
\eta
\end{array}\right)\right)=s\left(\begin{array}{c}
\xi \\
-\eta
\end{array}\right), \forall \xi, \eta \in H_{\mathbf{R}} .
$$

It is straightforward to check that $\beta$ commutes with the modular action $\widetilde{\sigma}, \beta^{2}=\mathrm{Id}, \beta_{\mid \mathcal{M}}=$ $\operatorname{Id}_{\mid \mathcal{M}}$ and $\beta \alpha_{s}=\alpha_{-s} \beta, \forall s \in \mathbf{R}$. Since $\left(\alpha_{s}\right)$ and $\beta$ commute with the modular action $\widetilde{\sigma}$, one may extend $\left(\alpha_{s}\right)$ and $\beta$ to $\widetilde{M}$ by $\alpha_{s \mid L(\mathbf{R})}=\operatorname{Id}_{L(\mathbf{R})}$, for every $s \in \mathbf{R}$ and $\beta_{\mid L(\mathbf{R})}=\operatorname{Id}_{L(\mathbf{R})}$. Moreover $\left(\alpha_{s}, \beta\right)$ preserves the semifinite trace $\widehat{T r}$. Let's summarize what we have done so far:

Proposition 3.1. The $\widetilde{T r}$-preserving deformation $\left(\alpha_{s}, \beta\right)$ defined on $\widetilde{M}$ is s-malleable:

(1) $\alpha_{s \mid L(\mathbf{R})}=\operatorname{Id}_{L(\mathbf{R})}$, for every $s \in \mathbf{R}$ and $\alpha_{1}\left(x *_{L(\mathbf{R})} 1\right)=1 *_{L(\mathbf{R})} x$, for every $x \in M$.

(2) $\beta^{2}=\operatorname{Id}$ and $\beta_{\mid M}=\operatorname{Id}_{\mid M}$.

(3) $\beta \alpha_{s}=\alpha_{-s} \beta$, for every $s \in \mathbf{R}$.

Denote by $E_{M}: \widetilde{M} \rightarrow M$ the canonical trace-preserving conditional expectation. Since $\widetilde{\operatorname{Tr}}_{\mid M}=\operatorname{Tr}$, we will simply denote by $\operatorname{Tr}$ the semifinite trace on $\widetilde{M}$. Remind that the smalleable deformation $\left(\alpha_{s}, \beta\right)$ automatically features a certain transversality property.

Proposition 3.2 (Popa, [19]). We have the following:

$$
\left\|x-\alpha_{2 s}(x)\right\|_{2, \operatorname{Tr}} \leq 2\left\|\alpha_{s}(x)-E_{M}\left(\alpha_{s}(x)\right)\right\|_{2, \operatorname{Tr}}, \forall x \in L^{2}(M, \operatorname{Tr}), \forall s>0 .
$$

The next proposition refered in the Introduction as the spectral gap property was first proved by Popa in [20] for free products of finite von Neumann algebras. We will need the following straightforward generalization:

Proposition 3.3 ([5]). We keep the same notation as before. Let $q \in M$ be a non-zero projection such that $\operatorname{Tr}(q)<\infty$. Let $Q \subset q M q$ be a von Neumann subalgebra with no amenable direct summand. Then for any free ultrafilter $\omega$ on $\mathbf{N}$, we have $Q^{\prime} \cap(q \widetilde{M} q)^{\omega} \subset(q M q)^{\omega}$. 
Let $q \in M$ be a non-zero projection such that $\operatorname{Tr}(q)<\infty$. Note that $\operatorname{Tr}(q \cdot q) / \operatorname{Tr}(q)$ is a finite trace on $q \widetilde{M} q$. If $Q \subset q M q$ has no amenable direct summand, then for any $\varepsilon>0$, there exist $\delta>0$ and a finite subset $F \subset \mathcal{U}(Q)$ such that for any $x \in(q \widetilde{M} q)_{1}$ (the unit ball w.r.t. the operator norm),

$$
\|u x-x u\|_{2, \operatorname{Tr}}<\delta, \forall u \in F \Longrightarrow\left\|x-E_{q M q}(x)\right\|_{2, \operatorname{Tr}}<\varepsilon .
$$

We will simply denote $u x-x u$ by $[u, x]$.

3.2. Semisolidity of the continuous core. The following theorem is in some ways a reminiscence of a result of Ioana, Peterson \& Popa, namely Theorem 4.3 of 14 and also Theorem 4.2 of [5]. The deformation/spectral gap rigidity strategy enables us to locate inside the core $M$ of a free Araki-Woods factor the position of subalgebras $A \subset M$ with a large relative commutant $A^{\prime} \cap M$.

Theorem 3.4. Let $\mathcal{M}=\Gamma\left(H_{\mathbf{R}}, U_{t}\right)^{\prime \prime}$ be a free Araki-Woods factor and $M=\mathcal{M} \rtimes_{\sigma} \mathbf{R}$ be its continuous core. Let $q \in L(\mathbf{R}) \subset M$ be a non-zero projection such that $\operatorname{Tr}(q)<$ $\infty$. Let $Q \subset q M q$ be a von Neumann subalgebra with no amenable direct summand. Then $Q^{\prime} \cap q M q \preceq_{M} L(\mathbf{R})$.

Corollary 3.5. Let $\mathcal{M}=\Gamma\left(H_{\mathbf{R}}, U_{t}\right)^{\prime \prime}$ be a free Araki-Woods factor of type $\mathrm{III}_{1}$. Then the continuous core $M=\mathcal{M} \rtimes_{\sigma} \mathbf{R}$ is a semisolid $\mathrm{II}_{\infty}$ factor. Since $M$ is non-amenable, $M$ is always a prime factor.

Proof of Theorem 3.4. Let $q \in L(\mathbf{R})$ be a non-zero projection such that $\operatorname{Tr}(q)<\infty$. Let $Q \subset q M q$ be a von Neumann subalgebra with no amenable direct summand. Denote by $Q_{0}=$ $Q^{\prime} \cap q M q$. We keep the notation introduced previously and regard $M \subset \widetilde{M}=M_{1} *_{L(\mathbf{R})} M_{2}$ via the identification of $M$ with $M_{1}$. Remind that $\alpha_{s \mid L(\mathbf{R})}=\operatorname{Id}_{L(\mathbf{R})}$, for every $s \in \mathbf{R}$. In particular $\alpha_{s}(q)=q$, for every $s \in \mathbf{R}$.

Step (1) : Using the spectral gap condition and the transversality property of $\left(\alpha_{t}, \beta\right)$ to find $t>0$ and a non-zero intertwiner $v$ between Id and $\alpha_{t}$.

Let $\varepsilon=\frac{1}{4}\|q\|_{2, \operatorname{Tr}}$. We know that there exist $\delta>0$ and a finite subset $F \subset \mathcal{U}(Q)$, such that for every $x \in(q \widetilde{M} q)_{1}$,

$$
\|[x, u]\|_{2, \operatorname{Tr}} \leq \delta, \forall u \in F \Longrightarrow\left\|x-E_{q M q}(x)\right\|_{2, \operatorname{Tr}} \leq \varepsilon .
$$

Since $\alpha_{t} \rightarrow$ Id pointwise $*$-strongly, as $t \rightarrow 0$, and since $F$ is a finite subset of $Q \subset q M q$, we may choose $t=1 / 2^{k}$ small enough $(k \geq 1)$ such that

$$
\max \left\{\left\|u-\alpha_{t}(u)\right\|_{2, \operatorname{Tr}}: u \in F\right\} \leq \frac{\delta}{2} .
$$

For every $x \in\left(Q_{0}\right)_{1}$ and every $u \in F \subset Q$, since $[u, x]=0$, we have

$$
\begin{aligned}
\left\|\left[\alpha_{t}(x), u\right]\right\|_{2, \operatorname{Tr}} & =\left\|\left[\alpha_{t}(x), u-\alpha_{t}(u)\right]\right\|_{2, \operatorname{Tr}} \\
& \leq 2\left\|u-\alpha_{t}(u)\right\|_{2, \operatorname{Tr}} \\
& \leq \delta .
\end{aligned}
$$

Consequently, we get for every $x \in\left(Q_{0}\right)_{1},\left\|\alpha_{t}(x)-E_{q M q}\left(\alpha_{t}(x)\right)\right\|_{2, \operatorname{Tr}} \leq \varepsilon$. Using Proposition 3.2, we obtain for every $x \in\left(Q_{0}\right)_{1}$

$$
\left\|x-\alpha_{s}(x)\right\|_{2, \operatorname{Tr}} \leq \frac{1}{2}\|q\|_{2, \operatorname{Tr}},
$$


where $s=2 t$. Thus, for every $u \in \mathcal{U}\left(Q_{0}\right)$, we have

$$
\begin{aligned}
\left\|u^{*} \alpha_{s}(u)-q\right\|_{2, \operatorname{Tr}} & =\left\|u^{*}\left(\alpha_{s}(u)-u\right)\right\|_{2, \operatorname{Tr}} \\
& \leq\left\|u-\alpha_{s}(u)\right\|_{2, \operatorname{Tr}} \\
& \leq \frac{1}{2}\|q\|_{2, \operatorname{Tr}} .
\end{aligned}
$$

Denote by $\mathcal{C}=\overline{\mathrm{CO}}^{w}\left\{u^{*} \alpha_{s}(u): u \in \mathcal{U}\left(Q_{0}\right)\right\} \subset q L^{2}(\widetilde{M}) q$ the ultraweak closure of the convex hull of all $u^{*} \alpha_{s}(u)$, where $u \in \mathcal{U}\left(Q_{0}\right)$. Denote by $a$ the unique element in $\mathcal{C}$ of minimal $\|\cdot\|_{2, \operatorname{Tr}^{-}}$ norm. Since $\|a-q\|_{2, \operatorname{Tr}} \leq 1 / 2\|q\|_{2, \operatorname{Tr}}$, necessarily $a \neq 0$. Fix $u \in \mathcal{U}\left(Q_{0}\right)$. Since $u^{*} a \alpha_{s}(u) \in \mathcal{C}$ and $\left\|u^{*} a \alpha_{s}(u)\right\|_{2, \operatorname{Tr}}=\|a\|_{2, \operatorname{Tr}}$, necessarily $u^{*} a \alpha_{s}(u)=a$. Taking $v=\operatorname{pol}(a)$ the polar part of $a$, we have found a non-zero partial isometry $v \in q \widetilde{M} q$ such that

$$
x v=v \alpha_{s}(x), \forall x \in Q_{0} .
$$

Step (2) : Proving $Q_{0} \preceq_{M} L(\mathbf{R})$ using the malleability of $\left(\alpha_{t}, \beta\right)$. By contradiction, assume $Q_{0} \npreceq_{M} L(\mathbf{R})$. The first task is to lift Equation (5) to $s=1$. Note that it is enough to find a non-zero partial isometry $w \in q \widetilde{M} q$ such that

$$
x w=w \alpha_{2 s}(x), \forall x \in Q_{0} .
$$

Indeed, by induction we can go till $s=1$ (because $s=1 / 2^{k-1}$ ). Remind that $\beta(z)=z$, for every $z \in M$. Note that $v v^{*} \in Q_{0}^{\prime} \cap q \widetilde{M} q$. Since $Q_{0} \npreceq M L(\mathbf{R})$, we know from Theorem 2.4 in [5] that $Q_{0}^{\prime} \cap q \widetilde{M} q \subset q M q$. In particular, $v v^{*} \in q M q$. Set $w=\alpha_{s}\left(\beta\left(v^{*}\right) v\right)$. Then,

$$
\begin{aligned}
w w^{*} & =\alpha_{s}\left(\beta\left(v^{*}\right) v v^{*} \beta(v)\right) \\
& =\alpha_{s}\left(\beta\left(v^{*}\right) \beta\left(v v^{*}\right) \beta(v)\right) \\
& =\alpha_{s} \beta\left(v^{*} v\right) \neq 0 .
\end{aligned}
$$

Hence, $w$ is a non-zero partial isometry in $q \widetilde{M} q$. Moreover, for every $x \in Q_{0}$,

$$
\begin{aligned}
w \alpha_{2 s}(x) & =\alpha_{s}\left(\beta\left(v^{*}\right) v \alpha_{s}(x)\right) \\
& =\alpha_{s}\left(\beta\left(v^{*}\right) x v\right) \\
& =\alpha_{s}\left(\beta\left(v^{*} x\right) v\right) \\
& =\alpha_{s}\left(\beta\left(\alpha_{s}(x) v^{*}\right) v\right) \\
& =\alpha_{s} \beta \alpha_{s}(x) \alpha_{s}\left(\beta\left(v^{*}\right) v\right) \\
& =\beta(x) w \\
& =x w .
\end{aligned}
$$

Since by induction, we can go till $s=1$, we have found a non-zero partial isometry $v \in q \widetilde{M} q$ such that

$$
x v=v \alpha_{1}(x), \forall x \in Q_{0} .
$$

Note that $v^{*} v \in \alpha_{1}\left(Q_{0}\right)^{\prime} \cap q M q$. Moreover, since $\alpha_{1}: q \widetilde{M} q \rightarrow q \widetilde{M} q$ is a $*$-automorphism, and $Q_{0} \npreceq_{M} L(\mathbf{R})$, Theorem 2.4 in [5] gives

$$
\begin{aligned}
\alpha_{1}\left(Q_{0}\right)^{\prime} \cap q \widetilde{M} q & =\alpha_{1}\left(Q_{0}^{\prime} \cap q \widetilde{M} q\right) \\
& \subset \alpha_{1}(q M q) .
\end{aligned}
$$

Hence $v^{*} v \in \alpha_{1}(q M q)$.

Since $Q_{0} \npreceq_{M} L(\mathbf{R})$, we know that there exists a sequence of unitaries $\left(u_{k}\right)$ in $Q_{0}$ such that $E_{L(\mathbf{R})}\left(x^{*} u_{k} y\right) \rightarrow 0$ strongly, as $k \rightarrow \infty$, for any $x, y \in q M$. We need to go further and prove the following: 
Claim 3.6. $\forall a, b \in q \widetilde{M} q,\left\|E_{M_{2}}\left(a^{*} u_{k} b\right)\right\|_{2, \operatorname{Tr}} \rightarrow 0$, as $k \rightarrow \infty$.

Proof of Claim 3.6. Let $a, b \in(\widetilde{M})_{1}$ be either elements in $L(\mathbf{R})$ or reduced words with letters alternating from $M_{1} \ominus L(\mathbf{R})$ and $M_{2} \ominus L(\mathbf{R})$. Write $b=y b^{\prime}$ with

- $y=b$ if $b \in L(\mathbf{R})$;

- $y=1$ if $b$ is a reduced word beginning with a letter from $M_{2} \ominus L(\mathbf{R})$;

- $y=$ the first letter of $b$ otherwise.

Note that either $b^{\prime}=1$ or $b^{\prime}$ is a reduced word beginning with a letter from $M_{2} \ominus L(\mathbf{R})$. Likewise write $a=a^{\prime} x$ with

- $x=a$ if $x \in L(\mathbf{R})$;

- $x=1$ if $a$ is a reduced word ending with a letter from $M_{2} \ominus L(\mathbf{R})$;

- $x=$ the last letter of $a$ otherwise.

Either $a^{\prime}=1$ or $a^{\prime}$ is a reduced word ending with a letter from $M_{2} \ominus L(\mathbf{R})$. For any $z \in Q_{0} \subset M_{1}, x z y-E_{L(\mathbf{R})}(x z y) \in M_{1} \ominus L(\mathbf{R})$, so that

$$
E_{M_{2}}(a z b)=E_{M_{2}}\left(a^{\prime} E_{L(\mathbf{R})}(x z y) b^{\prime}\right) .
$$

Since $E_{L(\mathbf{R})}\left(x u_{k} y\right) \rightarrow 0$ strongly, as $k \rightarrow \infty$, it follows that $E_{M_{2}}\left(a u_{k} b\right) \rightarrow 0$ strongly, as $k \rightarrow$ $\infty$, as well. Thus, in the finite von Neumann algebra $q \widetilde{M} q$, we get $\left\|q E_{M_{2}}\left(a u_{k} b\right) q\right\|_{2, \operatorname{Tr}} \rightarrow 0$, as $k \rightarrow \infty$.

Note that

$$
\mathcal{A}:=\operatorname{span}\left\{L(\mathbf{R}),\left(M_{i_{1}} \ominus L(\mathbf{R})\right) \cdots\left(M_{i_{n}} \ominus L(\mathbf{R})\right): n \geq 1, i_{1} \neq \cdots \neq i_{n}\right\}
$$

is a unital $*$-strongly dense $*$-subalgebra of $\widetilde{M}$. What we have shown so far is that for any $a, b \in \mathcal{A},\left\|q E_{M_{2}}\left(a u_{k} b\right) q\right\|_{2, \operatorname{Tr}} \rightarrow 0$, as $k \rightarrow \infty$. Let now $a, b \in(\widetilde{M})_{1}$. By Kaplansky density theorem, let $\left(a_{i}\right)$ and $\left(b_{j}\right)$ be sequences in $(\mathcal{A})_{1}$ such that $a_{i} \rightarrow a$ and $b_{j} \rightarrow b$ strongly. Recall that $\left(u_{k}\right)$ is a sequence in $Q_{0} \subset q \widetilde{M} q$. We have

$$
\begin{aligned}
\left\|q E_{M_{2}}\left(a u_{k} b\right) q\right\|_{2, \operatorname{Tr}} \leq & \left\|q E_{M_{2}}\left(a_{i} u_{k} b_{j}\right) q\right\|_{2, \operatorname{Tr}}+\left\|q E_{M_{2}}\left(a_{i} u_{k}\left(b-b_{j}\right)\right) q\right\|_{2, \operatorname{Tr}} \\
& +\left\|q E_{M_{2}}\left(\left(a-a_{i}\right) u_{k} b_{j}\right) q\right\|_{2, \operatorname{Tr}}+\left\|q E_{M_{2}}\left(\left(a-a_{i}\right) u_{k}\left(b-b_{j}\right)\right) q\right\|_{2, \operatorname{Tr}} \\
\leq & \left\|q E_{M_{2}}\left(a_{i} u_{k} b_{j}\right) q\right\|_{2, \operatorname{Tr}}+\left\|q a_{i} u_{k}\left(b-b_{j}\right) q\right\|_{2, \operatorname{Tr}} \\
& +\left\|q\left(a-a_{i}\right) u_{k} b_{j} q\right\|_{2, \operatorname{Tr}}+\left\|q\left(a-a_{i}\right) u_{k}\left(b-b_{j}\right) q\right\|_{2, \operatorname{Tr}} \\
\leq & \left\|q E_{M_{2}}\left(a_{i} u_{k} b_{j}\right) q\right\|_{2, \operatorname{Tr}}+3\left\|\left(b-b_{j}\right) q\right\|_{2, \operatorname{Tr}}+\left\|q\left(a-a_{i}\right) q\right\|_{2, \operatorname{Tr}}
\end{aligned}
$$

Fix $\varepsilon>0$. Since $a_{i} \rightarrow a$ and $b_{j} \rightarrow b$ strongly, let $i_{0}, j_{0}$ large enough such that

$$
3\left\|\left(b-b_{j_{0}}\right) q\right\|_{2, \operatorname{Tr}}+\left\|q\left(a-a_{i_{0}}\right) q\right\|_{2, \operatorname{Tr}} \leq \varepsilon / 2 .
$$

Now let $k_{0} \in \mathbf{N}$ such that for any $k \geq k_{0}$,

$$
\left\|q E_{M_{2}}\left(a_{i_{0}} u_{k} b_{j_{0}}\right) q\right\|_{2, \operatorname{Tr}} \leq \varepsilon / 2 \text {. }
$$

We finally get $\left\|q E_{M_{2}}\left(a u_{k} b\right) q\right\|_{2, T r} \leq \varepsilon$, for any $k \geq k_{0}$, which finishes the proof of the claim.

We remind that for any $x \in Q_{0}, v^{*} x v=\alpha_{1}(x) v^{*} v$. Moreover, $v^{*} v \in \alpha_{1}(q M q) \subset q M_{2} q$. So, for any $x \in Q_{0}, v^{*} x v \in q M_{2} q$. Since $\alpha_{1}\left(u_{k}\right) \in \mathcal{U}\left(q M_{2} q\right)$, we get

$$
\left\|v^{*} v\right\|_{2, \operatorname{Tr}}=\left\|\alpha_{1}\left(u_{k}\right) v^{*} v\right\|_{2, \operatorname{Tr}}=\left\|E_{M_{2}}\left(\alpha_{1}\left(u_{k}\right) v^{*} v\right)\right\|_{2, \operatorname{Tr}}=\left\|E_{M_{2}}\left(v^{*} u_{k} v\right)\right\|_{2, \operatorname{Tr}} \rightarrow 0 .
$$

Thus $v=0$, which is a contradiction. 
Proof of Corollary 3.5. Let $q \in L(\mathbf{R})$ be a non-zero projection such that $\operatorname{Tr}(q)<\infty$. Denote by $N=q M q$ the corresponding $\mathrm{II}_{1}$ factor and by $\tau=\operatorname{Tr}(q \cdot q) / \operatorname{Tr}(q)$ the canonical trace on $N$. By contradiction, assume that $N$ is not semisolid. Then there exists $Q \subset N$ a nonamenable von Neumann subalgebra such that the relative commutant $Q^{\prime} \cap N$ is of type $\mathrm{II}_{1}$. Write $z \in \mathcal{Z}(Q)$ for the maximal projection such that $Q z$ is amenable. Then $1-z \neq 0$, the von Neumann algebra $Q(1-z)$ has no amenable direct summand and $\left(Q^{\prime} \cap N\right)(1-z)$ is still of type $\mathrm{II}_{1}$. We may choose a projection $q_{0} \in Q(1-z)$ such that $\tau\left(q_{0}\right)=1 / n$. Since $N$ is a $\mathrm{II}_{1}$ factor, we may replace $Q$ by $\mathbf{M}_{n}(\mathbf{C}) \otimes q_{0} Q q_{0}$, so that we may assume $Q \subset N$ has no amenable direct summand and $Q^{\prime} \cap N$ is still of type $\mathrm{II}_{1}$.

If we apply Theorem 3.4 it follows that $Q^{\prime} \cap N \preceq_{M} L(\mathbf{R})$. We get a contradiction because $Q^{\prime} \cap N$ is of type $\mathrm{II}_{1}$ and $L(\mathbf{R})$ is of type $\mathrm{I}$.

It follows from [28] that for any type $\mathrm{III}_{1}$ factor $\mathcal{M}$, if the continuous core $M=\mathcal{M} \rtimes_{\sigma} \mathbf{R}$ is full, then Connes' invariant $\tau(\mathcal{M})$ is the usual topology on $\mathbf{R}$. Let now $\mathcal{M}=\Gamma\left(H_{\mathbf{R}}, U_{t}\right)^{\prime \prime}$ be a free Araki-Woods factor associated with $\left(U_{t}\right)$ an almost periodic representation. Denote by $S_{U} \subset \mathbf{R}_{+}^{*}$ the (countable) subgroup generated by the point spectrum of $\left(U_{t}\right)$. Then $\tau(\mathcal{M})$ is strictly weaker than the usual topology. More precisely, the completion of $\mathbf{R}$ w.r.t. the topology $\tau(\mathcal{M})$ is the compact group $\widehat{S_{U}}$ dual of $S_{U}$ (see [6]). Therefore in this case, for any non-zero projection $q \in L(\mathbf{R})$ such that $\operatorname{Tr}(q)<\infty$, the $\mathrm{II}_{1}$ factor $q M q$ is semisolid, by Theorem 3.4, and has property $\Gamma$ of Murray \& von Neumann by the above remark.

3.3. Solidity of the continuous core under the assumption that $\left(U_{t}\right)$ is mixing. We start this subsection with the following observations. The solidity of the continuous core $M$ forces the centralizers on $\mathcal{M}$ to be amenable. Indeed, fix $\psi$ any f.n. state on $\mathcal{M}$. Assume that the continuous core $M \simeq \mathcal{M} \rtimes_{\sigma^{\psi}} \mathbf{R}$ is solid. Choose a non-zero projection $q \in L(\mathbf{R})$ such that $\operatorname{Tr}(q)<\infty$. Since $L(\mathbf{R}) q$ is diffuse in $q\left(\mathcal{M} \rtimes_{\sigma^{\psi}} \mathbf{R}\right) q$, its relative commutant must be amenable. In particular $\mathcal{M}^{\psi} \bar{\otimes} L(\mathbf{R}) q$ is amenable. Thus, $\mathcal{M}^{\psi}$ is amenable.

Note that if the orthogonal representation $\left(U_{t}\right)$ contains a $\frac{2 \pi}{|\log \lambda|}$-periodic subrepresentation $\left(V_{t}^{\lambda}\right), 0<\lambda<1$, of the form

$$
V_{t}^{\lambda}=\left(\begin{array}{cc}
\cos (t \log \lambda) & -\sin (t \log \lambda) \\
\sin (t \log \lambda) & \cos (t \log \lambda)
\end{array}\right)
$$

then the free Araki-Woods factor $\mathcal{M}=\Gamma\left(H_{\mathbf{R}}, U_{t}\right)^{\prime \prime}$ freely absorbs $L\left(\mathbf{F}_{\infty}\right)$ (see [32]):

$$
\left(\mathcal{M}, \varphi_{U}\right) *\left(L\left(\mathbf{F}_{\infty}\right), \tau\right) \cong\left(\mathcal{M}, \varphi_{U}\right) .
$$

In particular, the centralizer of the free quasi-free state $\mathcal{M}^{\varphi_{U}}$ is non-amenable since it contains $L\left(\mathbf{F}_{\infty}\right)$. Therefore, whenever $\left(U_{t}\right)$ contains a periodic subrepresentation of the form $\left(V_{t}^{\lambda}\right)$ for some $0<\lambda<1$, the continuous core of $\Gamma\left(H_{\mathbf{R}}, U_{t}\right)^{\prime \prime}$ is semisolid by Theorem 3.4 but can never be solid. However, when $\left(U_{t}\right)$ is assumed to be mixing, we get solidity of the continuous core. Indeed in that case, we can control the relative commutant $A^{\prime} \cap M$ of diffuse subalgebras $A \subset L(\mathbf{R}) \subset M$, where $M$ is the continuous core of the free Araki-Woods factor associated with $\left(U_{t}\right)$. Thus, the next theorem can be regarded as the analog of a result of Popa, namely Theorem 3.1 of [21] (see also Theorem D.4 in [36]).

Theorem 3.7. Let $\left(U_{t}\right)$ be a mixing orthogonal representation of $\mathbf{R}$ on the real Hilbert space $H_{\mathbf{R}}$. Denote by $\mathcal{M}=\Gamma\left(H_{\mathbf{R}}, U_{t}\right)^{\prime \prime}$ the corresponding free Araki-Woods factor and by $M=\mathcal{M} \rtimes_{\sigma} \mathbf{R}$ its continuous core. Let $k \geq 1$ and let $q \in \mathbf{M}_{k}(\mathbf{C}) \otimes L(\mathbf{R})$ be a non-zero projection such that $T:=\left(\operatorname{Tr}_{k} \otimes \operatorname{Tr}\right)(q)<\infty$. Write $L(\mathbf{R})^{T}:=q\left(\mathbf{M}_{k}(\mathbf{C}) \otimes L(\mathbf{R})\right) q$ and $M^{T}:=q\left(\mathbf{M}_{k}(\mathbf{C}) \otimes M\right) q$. Let $A \subset L(\mathbf{R})^{T}$ be a diffuse von Neumann subalgebra.

Then for any sub-bimodule ${ }_{A} H_{L(\mathbf{R})^{T}}$ of ${ }_{A} L^{2}\left(M^{T}\right)_{L(\mathbf{R})^{T}}$ such that $\operatorname{dim}\left(H_{L(\mathbf{R})^{T}}\right)<\infty$, one has $H \subset L^{2}\left(L(\mathbf{R})^{T}\right)$. In particular $A^{\prime} \cap M^{T} \subset L(\mathbf{R})^{T}$. 
Corollary 3.8. Let $\mathcal{M}=\Gamma\left(H_{\mathbf{R}}, U_{t}\right)^{\prime \prime}$ be a free Araki-Woods factor such that the orthogonal representation $\left(U_{t}\right)$ is mixing. Then the continuous core $M=\mathcal{M} \rtimes_{\sigma} \mathbf{R}$ is a solid $\mathrm{II}_{\infty}$ factor.

Proof of Theorem 3.7. As usual, denote by $\left(\lambda_{t}\right)$ the unitaries in $L(\mathbf{R})$ that implement the modular action $\sigma$ on $\mathcal{M}$. Let $\Phi: L^{\infty}(\mathbf{R}) \rightarrow L(\mathbf{R})$ be the Fourier Transform so that $\Phi\left(e^{i t \cdot}\right)=$ $\lambda_{t}$, for every $t \in \mathbf{R}$. Let $T>0$ and denote by $q=\Phi\left(\chi_{[0, T]}\right)$. Notice that $L^{\infty}(\mathbf{R}) \chi_{[0, T]} \cong$ $L^{\infty}[0, T]$ and that

$$
\operatorname{span}\left\{\sum_{k \in F} c_{k} e^{i \frac{2 \pi}{T} k \cdot} \chi_{[0, T]}: F \subset \mathbf{Z} \text { finite subset, } c_{k} \in \mathbf{C}, \forall k \in F\right\}
$$

is a unital $*$-strongly dense $*$-subalgebra of $L^{\infty}(\mathbf{R}) \chi_{[0, T]}$. Thus, using the isomorphism $\Phi$, we get that

$$
\mathcal{A}:=\operatorname{span}\left\{\sum_{k \in F} c_{k} \lambda_{\frac{2 \pi}{T} k} q: F \subset \mathbf{Z} \text { finite subset, } c_{k} \in \mathbf{C}, \forall k \in F\right\}
$$

is a unital $*$-strongly dense $*$-subalgebra of $L(\mathbf{R}) q$. Let $\left(u_{n}\right)$ be bounded sequence in $L(\mathbf{R}) q$ such that $u_{n} \rightarrow 0$ weakly, as $n \rightarrow \infty$, and $\left\|u_{n}\right\|_{\infty} \leq 1$, for every $n \in \mathbf{N}$. Using Kaplansky density theorem together with a standard diagonal process, choose a sequence $y_{n} \in \mathcal{A}$ such that $\left\|y_{n}\right\|_{\infty} \leq 1$, for every $n \in \mathbf{N}$, and $\left\|u_{n}-y_{n}\right\|_{2, \operatorname{Tr}} \rightarrow 0$, as $n \rightarrow \infty$. We will write $y_{n}=z_{n} q$ with

$$
z_{n}=\sum_{k \in F_{n}} c_{k, n} \lambda_{\frac{2 \pi}{T} k}
$$

where $F_{n} \subset \mathbf{Z}$ is finite, $c_{k, n} \in \mathbf{C}$, for any $k \in F_{n}$ and any $n \in \mathbf{N}$. Using the $T$-periodicity, we have for any $n \in \mathbf{N}$,

$$
\begin{aligned}
\left\|z_{n}\right\|_{\infty} & =\left\|\Phi^{-1}\left(z_{n}\right)\right\|_{\infty} \\
& =\underset{x \in \mathbf{R}}{\operatorname{ess} \sup }\left|\sum_{k \in F_{n}} c_{k, n} e^{i \frac{2 \pi}{T} k x}\right| \\
& =\underset{x \in[0, T]}{\operatorname{ess} \sup }\left|\sum_{k \in F_{n}} c_{k, n} e^{i \frac{2 \pi}{T} k x}\right| \\
& =\left\|\Phi^{-1}\left(z_{n}\right) \chi_{[0, T]}\right\|_{\infty} \\
& =\left\|y_{n}\right\|_{\infty} \leq 1 .
\end{aligned}
$$

Thus, the sequence $\left(z_{n}\right)$ is uniformly bounded.

The first step of the proof consists in proving the following:

$$
\left\|E_{L(\mathbf{R}) q}\left(a u_{n} b\right)\right\|_{2, \operatorname{Tr}} \rightarrow 0, \text { as } n \rightarrow \infty, \forall a, b \in q M q \cap \operatorname{ker}\left(E_{L(\mathbf{R}) q}\right) .
$$

Equivalently, we need to show that

$$
\left\|q E_{L(\mathbf{R})}\left(a u_{n} b\right) q\right\|_{2, \operatorname{Tr}} \rightarrow 0, \text { as } n \rightarrow \infty, \forall a, b \in \operatorname{ker}\left(E_{L(\mathbf{R})}\right) .
$$

The first step of the proof is now divided in three different claims that will lead to proving (77). First note that

$$
\mathcal{E}:=\operatorname{span}\left\{\sum_{t \in F} x_{t} \lambda_{t}: F \subset \mathbf{R} \text { finite subset, } x_{t} \in \mathcal{M} \text { with } \varphi\left(x_{t}\right)=0, \forall t \in F\right\}
$$

is $*$-strongly dense in $\operatorname{ker}\left(E_{L(\mathbf{R})}\right)$ by Kaplansky density theorem. We first prove the following:

Claim 3.9. If $\left\|q E_{L(\mathbf{R})}\left(x u_{n} y\right) q\right\|_{2, T r} \rightarrow 0$, as $n \rightarrow \infty, \forall x, y \in \mathcal{M}$ with $\varphi(x)=\varphi(y)=0$, then (17) is satisfied. 
Proof of Claim [3.9. Assume $\left\|q E_{L(\mathbf{R})}\left(x u_{n} y\right) q\right\|_{2, \operatorname{Tr}} \rightarrow 0$, as $n \rightarrow \infty, \forall x, y \in \mathcal{M}$ with $\varphi(x)=$ $\varphi(y)=0$. First take $a \in \mathcal{E}$ that we write $a=\sum_{s \in F} x_{s} \lambda_{s}$, with $F \subset \mathbf{R}$ finite subset, such that $x_{s} \in \mathcal{M}, \varphi\left(x_{s}\right)=0$, for every $s \in F$. Then take $b \in \operatorname{ker}\left(E_{L(\mathbf{R})}\right)$ and let $\left(b_{j}\right)_{j \in J}$ be a sequence in $\mathcal{E}$ such that $b-b_{j} \rightarrow 0 *$-strongly, as $j \rightarrow \infty$. Since $\left\|u_{n}\right\|_{\infty} \leq 1$, we get for any $n \in \mathbf{N}$ and any $j \in J$,

$$
\begin{aligned}
\left\|q E_{L(\mathbf{R})}\left(a u_{n} b\right) q\right\|_{2, \operatorname{Tr}} & \leq\left\|q E_{L(\mathbf{R})}\left(a u_{n} b_{j}\right) q\right\|_{2, \operatorname{Tr}}+\left\|q E_{L(\mathbf{R})}\left(a u_{n}\left(b-b_{j}\right)\right) q\right\|_{2, \operatorname{Tr}} \\
& \leq\left\|q E_{L(\mathbf{R})}\left(a u_{n} b_{j}\right) q\right\|_{2, \operatorname{Tr}}+\|a\|_{\infty}\left\|\left(b-b_{j}\right) q\right\|_{2, \operatorname{Tr}}
\end{aligned}
$$

Fix $\varepsilon>0$. Since $b-b_{j} \rightarrow 0 *$-strongly, as $j \rightarrow \infty$, fix $j_{0} \in J$ such that $\|a\|_{\infty}\left\|\left(b-b_{j_{0}}\right) q\right\|_{2, \operatorname{Tr}} \leq$ $\varepsilon / 2$. Write $b_{j_{0}}=\sum_{t \in F^{\prime}} y_{t} \lambda_{t}$, with $F^{\prime} \subset \mathbf{R}$ finite subset, such that $y_{t} \in \mathcal{M}, \varphi\left(y_{t}\right)=0$, for every $t \in F^{\prime}$. Therefore, for any $n \in \mathbf{N}$,

$$
\begin{aligned}
\left\|q E_{L(\mathbf{R})}\left(a u_{n} b_{j_{0}}\right) q\right\|_{2, \operatorname{Tr}} & \leq \sum_{(s, t) \in F \times F^{\prime}}\left\|q E_{L(\mathbf{R})}\left(x_{s} \lambda_{s} u_{n} y_{t} \lambda_{t}\right) q\right\|_{2, \operatorname{Tr}} \\
& =\sum_{(s, t) \in F \times F^{\prime}}\left\|\lambda_{s} q E_{L(\mathbf{R})}\left(\sigma_{-s}\left(x_{s}\right) u_{n} y_{t}\right) q \lambda_{t}\right\|_{2, \operatorname{Tr}} \\
& =\sum_{(s, t) \in F \times F^{\prime}}\left\|q E_{L(\mathbf{R})}\left(\sigma_{-s}\left(x_{s}\right) u_{n} y_{t}\right) q\right\|_{2, \operatorname{Tr}} .
\end{aligned}
$$

Since $\varphi\left(\sigma_{-s}\left(x_{s}\right)\right)=\varphi\left(y_{t}\right)=0$, for any $(s, t) \in F \times F^{\prime}$, using the assumption of the claim, there exists $n_{0} \in \mathbf{N}$ large enough such that for any $n \geq n_{0},\left\|q E_{L(\mathbf{R})}\left(a u_{n} b_{j_{0}}\right) q\right\|_{2, \operatorname{Tr}} \leq \varepsilon / 2$. Thus, for any $n \geq n_{0},\left\|q E_{L(\mathbf{R})}\left(a u_{n} b\right) q\right\|_{2, T r} \leq \varepsilon$. This proves that for any $a \in \mathcal{E}$ and any $b \in \operatorname{ker}\left(E_{L(\mathbf{R})}\right),\left\|q E_{L(\mathbf{R})}\left(a u_{n} b\right) q\right\|_{2, \operatorname{Tr}} \rightarrow 0$, as $n \rightarrow \infty$. If we do the same thing by approximating $a \in \operatorname{ker}\left(E_{L(\mathbf{R})}\right)$ with elements in $\mathcal{E}$, using the fact that $u_{n} \in(L(\mathbf{R}) q)_{1}$, we finally get the claim.

We now replace the sequence $\left(u_{n}\right)$ by $\left(z_{n}\right)$, use the mixing property of the modular action $\sigma$ and prove the following:

Claim 3.10. $\forall a, b \in(\mathcal{M})_{1}$ with $\varphi(a)=\varphi(b)=0,\left\|q E_{L(\mathbf{R})}\left(a z_{n} b\right) q\right\|_{2, \operatorname{Tr}} \rightarrow 0$, as $n \rightarrow \infty$.

Proof of Claim 3.10. Fix $a, b \in(\mathcal{M})_{1}$ such that $\varphi(a)=\varphi(b)=0$. Fix $\varepsilon>0$. For any $n \in \mathbf{N}$, we have

$$
\begin{aligned}
\left\|q E_{L(\mathbf{R})}\left(a z_{n} b\right) q\right\|_{2, \operatorname{Tr}}^{2} & =\left\|\sum_{k \in F_{n}} c_{k, n} \varphi\left(a \sigma_{\frac{2 \pi}{T} k}(b)\right) \lambda_{\frac{2 \pi}{T} k} q\right\|_{2, \operatorname{Tr}}^{2} \\
& =\operatorname{Tr}(q) \sum_{k \in F_{n}}\left|c_{k, n}\right|^{2}\left|\varphi\left(a \sigma_{\frac{2 \pi}{T} k}(b)\right)\right|^{2} .
\end{aligned}
$$

Moreover for any $n \in \mathbf{N}$,

$$
\operatorname{Tr}(q) \sum_{k \in F_{n}}\left|c_{k, n}\right|^{2}=\left\|z_{n} q\right\|_{2, \operatorname{Tr}}^{2} \leq \operatorname{Tr}(q)\left\|z_{n} q\right\|_{\infty}^{2} \leq T .
$$

Since the modular group $\sigma$ is $\varphi$-mixing (because $\left(U_{t}\right)$ is assumed to be mixing), there exists a finite subset $K \subset \mathbf{Z}$ such that for any $k \in \mathbf{Z} \backslash K,\left|\varphi\left(a \sigma_{\frac{2 \pi}{T} k}(b)\right)\right| \leq \varepsilon / \sqrt{2 T}$. Thus,

$$
\left\|q E_{L(\mathbf{R})}\left(a z_{n} b\right) q\right\|_{2, \operatorname{Tr}} \leq\left\|\sum_{k \in K \cap F_{n}} c_{k, n} \lambda_{\frac{2 \pi}{T} k} q\right\|_{2, \operatorname{Tr}}+\varepsilon / 2 .
$$


Since $u_{n}-z_{n} q \rightarrow 0$ strongly and $u_{n} \rightarrow 0$ weakly, as $n \rightarrow \infty$, it follows that $z_{n} q \rightarrow 0$ weakly, as $n \rightarrow \infty$. In particular there exists $n_{0}$ large enough such that for any $n \geq n_{0}$, for any $k \in K \cap F_{n},\left|c_{k, n}\right| \leq \varepsilon /\left(2|K|\|q\|_{2, \operatorname{Tr}}\right)$. Thus, for any $n \geq n_{0}$,

$$
\left\|q E_{L(\mathbf{R})}\left(a z_{n} b\right) q\right\|_{2, \operatorname{Tr}} \leq \varepsilon / 2+\varepsilon / 2=\varepsilon .
$$

This proves that $\left\|q E_{L(\mathbf{R})}\left(a z_{n} b\right) q\right\|_{2, \operatorname{Tr}} \rightarrow 0$, as $n \rightarrow \infty$.

The last claim consists in going back to the sequence $\left(u_{n}\right)$ and proving the following:

Claim 3.11. $\forall a, b \in(\mathcal{M})_{1}$ with $\varphi(a)=\varphi(b)=0,\left\|q E_{L(\mathbf{R})}\left(a u_{n} b\right) q\right\|_{2, \operatorname{Tr}} \rightarrow 0$, as $n \rightarrow \infty$.

Proof of Claim 3.11. Applying once more Kaplansky density theorem, we can find a sequence $\left(q_{i}\right)_{i \in I}$ in $L(\mathbf{R})$ such that

- $q_{i}=\sum_{t \in F_{i}} d_{t} \lambda_{t}$, with $F_{i} \subset \mathbf{R}$ finite subset, $d_{t} \in \mathbf{C}$, for any $t \in F_{i}$ and for any $i \in I$;

- $\left\|q_{i}\right\|_{\infty} \leq 1$, for any $i \in I$;

- $q-q_{i} \rightarrow 0$ *-strongly, as $i \rightarrow \infty$.

Fix now $a, b \in(\mathcal{M})_{1}$ such that $\varphi(a)=\varphi(b)=0$. Using the fact that

$$
\|a\|_{\infty},\|b\|_{\infty},\|q\|_{\infty},\left\|z_{n}\right\|_{\infty} \leq 1, \forall n \in \mathbf{N}
$$

we get for any $n \in \mathbf{N}$ and any $i \in I$,

$$
\begin{aligned}
\left\|q E_{L(\mathbf{R})}\left(a u_{n} b\right) q\right\|_{2, \operatorname{Tr}} \leq & \left\|q E_{L(\mathbf{R})}\left(a\left(u_{n}-z_{n} q\right) b\right) q\right\|_{2, \operatorname{Tr}}+\left\|q E_{L(\mathbf{R})}\left(a z_{n} q b\right) q\right\|_{2, \operatorname{Tr}} \\
\leq & \left\|u_{n}-z_{n} q\right\|_{2, \operatorname{Tr}}+\left\|q E_{L(\mathbf{R})}\left(a z_{n}\left(q-q_{i}\right) b\right) q\right\|_{2, \operatorname{Tr}} \\
& +\left\|q E_{L(\mathbf{R})}\left(a z_{n} q_{i} b\right) q\right\|_{2, \operatorname{Tr}} \\
\leq & \left\|u_{n}-z_{n} q\right\|_{2, \operatorname{Tr}}+\left\|\left(q-q_{i}\right) b q\right\|_{2, \operatorname{Tr}} \\
& +\sum_{t \in F_{i}}\left|d_{t}\right|\left\|q E_{L(\mathbf{R})}\left(a z_{n} \sigma_{t}(b)\right) \lambda_{t} q\right\|_{2, \operatorname{Tr}} \\
\leq & \left\|u_{n}-z_{n} q\right\|_{2, \operatorname{Tr}}+\left\|\left(q-q_{i}\right) b q\right\|_{2, \operatorname{Tr}} \\
& +\sum_{t \in F_{i}}\left|d_{t}\right|\left\|q E_{L(\mathbf{R})}\left(a z_{n} \sigma_{t}(b)\right) q\right\|_{2, \operatorname{Tr}} .
\end{aligned}
$$

Since $q-q_{i} \rightarrow 0 *$-strongly, as $i \rightarrow \infty$, it follows that $\left\|\left(q-q_{i}\right) b q\right\|_{2, \operatorname{Tr}} \rightarrow 0$, as $i \rightarrow \infty$. Fix $\varepsilon>0$. Then, take $i_{0} \in I$ such that $\left\|\left(q-q_{i_{0}}\right) b q\right\|_{2, \operatorname{Tr}} \leq \varepsilon / 3$. Since $\left\|u_{n}-z_{n} q\right\|_{2, \operatorname{Tr}} \rightarrow 0$, as $n \rightarrow \infty$ and using Claim 3.10, we may choose $n_{0}$ large enough such that for any $n \geq n_{0}$,

$$
\begin{aligned}
\left\|u_{n}-z_{n} q\right\|_{2, \operatorname{Tr}} & \leq \varepsilon / 3 \\
\sum_{t \in F_{i_{0}}}\left|d_{t}\right|\left\|q E_{L(\mathbf{R})}\left(a z_{n} \sigma_{t}(b)\right) q\right\|_{2, \operatorname{Tr}} & \leq \varepsilon / 3 .
\end{aligned}
$$

Consequently, for any $n \geq n_{0}$, we get $\left\|q E_{L(\mathbf{R})}\left(a u_{n} b\right) q\right\|_{2, \operatorname{Tr}} \leq \varepsilon$. Therefore, we have proven $\left\|q E_{L(\mathbf{R})}\left(a u_{n} b\right) q\right\|_{2, \operatorname{Tr}} \rightarrow 0$, as $n \rightarrow \infty$.

Thanks to Claims 3.9 and 3.11, it is then clear that (7) is satisfied. This finishes the first step of the proof.

The last step of the proof consists in using Theorem 2.8. Let $k \geq 1$ and $q \in \mathbf{M}_{k}(\mathbf{C}) \otimes L(\mathbf{R})$ be a non-zero projection such that $T:=\left(\operatorname{Tr}_{k} \otimes \operatorname{Tr}\right)(q)<\infty$. Since $\mathbf{M}_{k}(\mathbf{C}) \otimes M$ is a $\mathrm{II}_{\infty}$ factor, there exists a unitary $u \in \mathcal{U}\left(\mathbf{M}_{k}(\mathbf{C}) \otimes M\right)$ such that

$$
q=u\left(\begin{array}{ccc}
q_{0} & & 0 \\
& \ddots & \\
0 & & q_{0}
\end{array}\right) u^{*}
$$


where $q_{0}=\Phi\left(\chi_{[0, T / k]}\right) \in L(\mathbf{R})$. Using the spatiality of $\operatorname{Ad}(u)$ on $\mathbf{M}_{k}(\mathbf{C}) \otimes M$, we may assume without loss of generality that

$$
q=\left(\begin{array}{lll}
q_{0} & & 0 \\
& \ddots & \\
0 & & q_{0}
\end{array}\right)
$$

In particular, $q \in \mathbf{M}_{k}(\mathbf{C}) \otimes L(\mathbf{R}) q_{0}$. Define $M^{T}:=q\left(\mathbf{M}_{k}(\mathbf{C}) \otimes M\right) q$ and $L(\mathbf{R})^{T}:=q\left(\mathbf{M}_{k}(\mathbf{C}) \otimes\right.$ $L(\mathbf{R})) q$. Let $A \subset L(\mathbf{R})^{T}$ be a diffuse von Neumann subalgebra. Choose a sequence of unitaries $\left(u_{n}\right)$ in $A$ such that $u_{n} \rightarrow 0$ weakly, as $n \rightarrow \infty$. Thus, we can write $u_{n}=\left[u_{n}^{i, j}\right]_{i, j}$ where $u_{n}^{i, j} \in L(\mathbf{R}) q_{0}$ and $\left\|u_{n}^{i, j}\right\|_{\infty} \leq 1$, for any $n \in \mathbf{N}$ and any $i, j \in\{1, \ldots, k\}$. Moreover, $u_{n}^{i, j} \rightarrow 0$ weakly, as $n \rightarrow \infty$, in $L(\mathbf{R}) q_{0}$, for any $i, j \in\{1, \ldots, k\}$. Thus, using the first step of the proof, it becomes clear that the inclusion $L(\mathbf{R})^{T} \subset M^{T}$ is weakly mixing through $A$ in the sense of Definition 2.7. Thus, using Theorem 2.8, it follows that for any ${ }_{A} H_{L(\mathbf{R})^{T}}$ sub-bimodule of ${ }_{A} L^{2}\left(M^{T}\right)_{L(\mathbf{R})^{T}}$ such that $\operatorname{dim}\left(H_{L(\mathbf{R})^{T}}\right)<\infty$, one has $H \subset L^{2}\left(L(\mathbf{R})^{T}\right)$. In particular $A^{\prime} \cap M^{T} \subset L(\mathbf{R})^{T}$.

Proof of Corollary 3.8 . Let $q \in L(\mathbf{R})$ be a non-zero projection such that $\operatorname{Tr}(q)<\infty$. Denote by $N=q M q$ the corresponding $\mathrm{II}_{1}$ factor. By contradiction assume that $N$ is not solid. Then there exists a non-amenable von Neumann subalgebra $Q \subset N$ such that the relative commutant $Q^{\prime} \cap N$ is diffuse. Since $N$ is a $\mathrm{II}_{1}$ factor, using the same argument as in the proof of Corollary 3.5. we may assume that $Q$ has no amenable direct summand and $Q_{0}=Q^{\prime} \cap N$ is still diffuse.

Since $Q$ has no amenable direct summand, Theorem 3.4 yields $Q_{0} \preceq_{M} L(\mathbf{R})$. Thus using Theorem 2.9, we know that there exists a non-zero projection $p \in L(\mathbf{R})$ such that $\operatorname{Tr}(p)<$ $\infty$, and $Q_{0} \preceq_{e M e} L(\mathbf{R}) p$ where $e=p \vee q$. Consequently, there exist $n \geq 1$, a (possibly non-unital) *-homomorphism $\psi: Q_{0} \rightarrow \mathbf{M}_{n}(\mathbf{C}) \otimes L(\mathbf{R}) p$ and a non-zero partial isometry $v \in \mathbf{M}_{1, n}(\mathbf{C}) \otimes q M p$ such that

$$
x v=v \psi(x), \forall x \in Q_{0} .
$$

We moreover have

$$
v v^{*} \in Q_{0}^{\prime} \cap q M q \text { and } v^{*} v \in \psi\left(Q_{0}\right)^{\prime} \cap \psi(q)\left(\mathbf{M}_{n}(\mathbf{C}) \otimes p M p\right) \psi(q) .
$$

Write $Q_{1}=Q_{0}^{\prime} \cap q M q$ and notice that $Q \subset Q_{1}$. Since $\psi\left(Q_{0}\right)$ is diffuse and $v^{*} v \in \psi\left(Q_{0}\right)^{\prime} \cap$ $\psi(q)\left(\mathbf{M}_{n}(\mathbf{C}) \otimes p M p\right) \psi(q)$, Theorem 3.7 yields $v^{*} v \in \psi(q)\left(\mathbf{M}_{n}(\mathbf{C}) \otimes L(\mathbf{R}) p\right) \psi(q)$, so that we may assume $v^{*} v=\psi(q)$. For any $y \in Q_{1}$, and any $x \in Q_{0}$,

$$
\begin{aligned}
v^{*} y v \psi(x) & =v^{*} y x v \\
& =v^{*} x y v \\
& =\psi(x) v^{*} y v .
\end{aligned}
$$

Thus, $v^{*} Q_{1} v \subset \psi\left(Q_{0}\right)^{\prime} \cap v^{*} v\left(\mathbf{M}_{n}(\mathbf{C}) \otimes p M p\right) v^{*} v$. Since $\psi\left(Q_{0}\right)$ is diffuse, Theorem 3.7 yields $v^{*} Q_{1} v \subset v^{*} v\left(\mathbf{M}_{n}(\mathbf{C}) \otimes L(\mathbf{R}) p\right) v^{*} v$. Since $Q$ has no amenable direct summand and $Q \subset Q_{1}$ is a unital von Neumann subalgebra, it follows that $Q_{1}$ has no amenable direct summand either. Thus the von Neumann algebra $v v^{*} Q_{1} v v^{*}$ is non-amenable. $\operatorname{But} \operatorname{Ad}\left(v^{*}\right): v v^{*} M v v^{*} \rightarrow$ $v^{*} v\left(\mathbf{M}_{n}(\mathbf{C}) \otimes p M p\right) v^{*} v$ is a $*$-isomorphism and

$$
\operatorname{Ad}\left(v^{*}\right)\left(v v^{*} Q_{1} v v^{*}\right) \subset v^{*} v\left(\mathbf{M}_{n}(\mathbf{C}) \otimes L(\mathbf{R}) p\right) v^{*} v .
$$

Since $v^{*} v\left(\mathbf{M}_{n}(\mathbf{C}) \otimes L(\mathbf{R}) p\right) v^{*} v$ is of type I, hence amenable, we get a contradiction.

Since the left regular representation $\left(\lambda_{t}\right)$ of $\mathbf{R}$ acting on $L_{\mathbf{R}}^{2}(\mathbf{R}$, Lebesgue) is mixing, the continuous core $M$ of $\Gamma\left(L_{\mathbf{R}}^{2}(\mathbf{R}, \text { Lebesgue }), \lambda_{t}\right)^{\prime \prime}$ is solid. We partially retrieve a previous result of Shlyakhtenko [31] where he proved in this case that $M \cong L\left(\mathbf{F}_{\infty}\right) \bar{\otimes} \mathbf{B}\left(\ell^{2}\right)$, which is solid by 
[15]. We will give in the next section an example of a non-amenable solid $\mathrm{II}_{1}$ factor with full fundamental group which is not isomorphic to any interpolated free group factor $L\left(\mathbf{F}_{t}\right)$, for $1<t \leq \infty$.

Note that the mixing property of the representation $\left(U_{t}\right)$ is not a necessary condition for the solidity of the continuous core $M$. Indeed, take $U_{t}=\operatorname{Id} \oplus \lambda_{t}$ on $H_{\mathbf{R}}=\mathbf{R} \oplus L_{\mathbf{R}}^{2}$ (R, Lebesgue). Then $\left(U_{t}\right)$ is not mixing, but the continuous core $M$ of $\Gamma\left(H_{\mathbf{R}}, U_{t}\right)^{\prime \prime}$ is still isomorphic to $L\left(\mathbf{F}_{\infty}\right) \bar{\otimes} \mathbf{B}\left(\ell^{2}\right)[29$.

\section{EXAMPLes OF SOLID $\mathrm{II}_{1}$ FACTORS}

4.1. Probability measures on the real line and unitary representations of $\mathbf{R}$. Write $\lambda$ for the Lebesgue measure on the real line $\mathbf{R}$. Let $\mu$ be a symmetric (positive) probability measure on $\mathbf{R}$, i.e. $\mu(X)=\mu(-X)$, for any Borel subset $X \subset \mathbf{R}$. Consider the following unitary representation $\left(U_{t}^{\mu}\right)$ of $\mathbf{R}$ on $L^{2}(\mathbf{R}, \mu)$ given by:

$$
\left(U_{t}^{\mu} f\right)(x)=e^{i t x} f(x), \forall f \in L^{2}(\mathbf{R}, \mu), \forall t, x \in \mathbf{R} .
$$

Define the Hilbert subspace of $L^{2}(\mathbf{R}, \mu)$

$$
K_{\mathbf{R}}^{\mu}:=\left\{f \in L^{2}(\mathbf{R}, \mu): f(x)=\overline{f(-x)}, \forall x \in \mathbf{R}\right\} .
$$

Since $\mu$ is assumed to be symmetric, the restriction of the inner product to $K_{\mathbf{R}}$ is real-valued. Indeed, for any $f, g \in K_{\mathbf{R}}^{\mu}$,

$$
\begin{aligned}
\langle f, g\rangle & =\int_{\mathbf{R}} f(x) \overline{g(x)} d \mu(x) \\
& =\int_{\mathbf{R}} f(-x) \overline{g(-x)} d \mu(-x) \\
& =\int_{\mathbf{R}} \overline{f(x)} g(x) d \mu(x) \\
& =\overline{\langle f, g\rangle} .
\end{aligned}
$$

Moreover the representation $\left(U_{t}^{\mu}\right)$ leaves $K_{\mathbf{R}}^{\mu}$ globally invariant. Thus, $\left(U_{t}^{\mu}\right)$ restricted to $K_{\mathbf{R}}^{\mu}$ becomes an orthogonal representation. Define the Fourier Transform of the probability measure $\mu$ by:

$$
\widetilde{\mu}(t)=\int_{\mathbf{R}} e^{i t x} d \mu(x), \forall t \in \mathbf{R} .
$$

We shall identify $\widehat{\mathbf{R}}$ with $\mathbf{R}$ in the usual way, such that

$$
\widehat{f}(t)=\int_{\mathbf{R}} e^{i t x} f(x) d \lambda(x), \forall t \in \mathbf{R}, \forall f \in L^{1}(\mathbf{R}, \lambda) .
$$

Proposition 4.1. Let $\mu$ be a symmetric probability measure on $\mathbf{R}$. Then

$$
\left(U_{t}^{\mu}\right) \text { is mixing } \Longleftrightarrow \widetilde{\mu}(t) \rightarrow 0 \text {, as }|t| \rightarrow \infty .
$$

Proof. We prove both directions.

$\Longrightarrow$ Assume $\left(U_{t}^{\mu}\right)$ is mixing. Let $f=\mathbf{1}_{\mathbf{R}} \in L^{2}(\mathbf{R}, \mu)$ be the constant function equal to 1 . Then

$$
\begin{aligned}
\widetilde{\mu}(t) & =\int_{\mathbf{R}} e^{i t x} d \mu(x) \\
& =\left\langle U_{t}^{\mu} f, f\right\rangle \rightarrow 0, \text { as }|t| \rightarrow \infty .
\end{aligned}
$$


$\Longleftarrow$ Assume $\widetilde{\mu}(t) \rightarrow 0$, as $|t| \rightarrow \infty$. Let $f, g \in L^{2}(\mathbf{R}, \mu)$. Then $h:=f \bar{g} \in L^{1}(\mathbf{R}, \mu)$. Since the set $\left\{f \in \mathrm{C}_{0}(\mathbf{R}): \widehat{f} \in L^{1}(\mathbf{R}, \lambda)\right\}$ is dense in $L^{1}(\mathbf{R}, \mu)$, we may choose a sequence $\left(h_{n}\right)$ in $\mathrm{C}_{0}(\mathbf{R})$ such that $\left\|h-h_{n}\right\|_{L^{1}(\mathbf{R}, \mu)} \rightarrow 0$, as $n \rightarrow \infty$, and $\widehat{h}_{n} \in L^{1}(\mathbf{R}, \lambda)$, for any $n \in \mathbf{N}$. Define

$$
\begin{aligned}
\widetilde{h}(t) & =\int_{\mathbf{R}} e^{i t x} h(x) d \mu(x), \forall t \in \mathbf{R} \\
\widetilde{h}_{n}(t) & =\int_{\mathbf{R}} e^{i t x} h_{n}(x) d \mu(x), \forall t \in \mathbf{R}, \forall n \in \mathbf{N} .
\end{aligned}
$$

Since $\left\|h-h_{n}\right\|_{L^{1}(\mathbf{R}, \mu)} \rightarrow 0$, as $n \rightarrow \infty$, it follows that $\left\|\widetilde{h}-\widetilde{h}_{n}\right\|_{\infty} \rightarrow 0$, as $n \rightarrow \infty$. Since $\widehat{h}_{n} \in L^{1}(\mathbf{R}, \lambda)$, we know that

$$
h_{n}(x)=C \int_{\mathbf{R}} e^{-i x u} \widehat{h}_{n}(u) d \lambda(u), \forall x \in \mathbf{R},
$$

where $C$ is a universal constant that only depends on the normalization of the Lebesgue measure $\lambda$ on $\mathbf{R}$. Therefore, for any $t \in \mathbf{R}$ and any $n \in \mathbf{N}$,

$$
\begin{aligned}
\widetilde{h}_{n}(t) & =\int_{x \in \mathbf{R}} e^{i t x} h_{n}(x) d \mu(x) \\
& =C \int_{x \in \mathbf{R}}\left(\int_{u \in \mathbf{R}} e^{i(t-u) x} \widehat{h}_{n}(u) d \lambda(u)\right) d \mu(x) \\
& =C \int_{u \in \mathbf{R}} \widehat{h}_{n}(u)\left(\int_{x \in \mathbf{R}} e^{i(t-u) x} d \mu(x)\right) d \lambda(u) \\
& =C \int_{u \in \mathbf{R}} \widehat{h}_{n}(u) \widetilde{\mu}(t-u) d \lambda(u) \\
& =C\left(\widehat{h}_{n} * \widetilde{\mu}\right)(t)
\end{aligned}
$$

where $*$ is the convolution product. Since $\widetilde{\mu} \in \mathrm{C}_{0}(\mathbf{R})$ and $\widehat{h}_{n} \in L^{1}(\mathbf{R}, \lambda)$, it is easy to check that $\widehat{h}_{n} * \widetilde{\mu} \in \mathrm{C}_{0}(\mathbf{R})$. Consequently, $\widetilde{h}_{n} \in \mathrm{C}_{0}(\mathbf{R})$ and since $\left\|\widetilde{h}-\widetilde{h}_{n}\right\|_{\infty} \rightarrow 0$, as $n \rightarrow \infty$, it follows that $\widetilde{h} \in \mathrm{C}_{0}(\mathbf{R})$. But for any $t \in \mathbf{R}$,

$$
\begin{aligned}
\left\langle U_{t}^{\mu} f, g\right\rangle & =\int_{\mathbf{R}} e^{i t x} f(x) \overline{g(x)} d \mu(x) \\
& =\widetilde{h}(t) .
\end{aligned}
$$

Thus, the unitary representation $\left(U_{t}^{\mu}\right)$ is mixing.

For a measure $\nu$ on $\mathbf{R}$, define the measure class of $\nu$ by:

$$
\mathcal{C}_{\nu}:=\left\{\nu^{\prime}: \nu^{\prime} \text { is absolutely continuous w.r.t. } \nu\right\} .
$$

Definition 4.2. Let $\left(V_{t}\right)$ be a unitary representation of $\mathbf{R}$ on a separable Hilbert space $H$. Denote by $B$ the infinitesimal generator of $\left(V_{t}\right)$, i.e. $B$ is the positive, self-adjoint (possibly) unbounded operator on $H$ such that $V_{t}=B^{i t}$, for every $t \in \mathbf{R}$. We define the spectral measure of the representation $\left(V_{t}\right)$ as the spectral measure of the operator $B$ and denote it by $\mathcal{C}_{V}$.

The measure class $\mathcal{C}_{V}$ can also be defined as the smallest collection of all the measures $\nu$ on $\mathbf{R}$ such that:

(1) If $\nu \in \mathcal{C}_{V}$ and $\nu^{\prime}$ is absolutely continuous w.r.t. $\nu$, then $\nu^{\prime} \in \mathcal{C}_{V}$;

(2) For any unit vector $\eta \in H$, the probability measure associated with the positive definite function $t \mapsto\left\langle V_{t} \eta, \eta\right\rangle$ belongs to $\mathcal{C}_{V}$. 
Since $H$ is separable, there exists a measure $\nu$ that generates $\mathcal{C}_{V}$, i.e. $\mathcal{C}_{V}$ is the smallest collection of measures on $\mathbf{R}$ satisfying (1) and containing $\nu$. We will refer to this particular measure $\nu$ as the "spectral measure" of the representation $\left(V_{t}\right)$ and simply denote it by $\nu$.

Let $\mu$ be a symmetric probability measure on $\mathbf{R}$ and consider the unitary representation $\left(U_{t}^{\mu}\right)$ on $L^{2}(\mathbf{R}, \mu)$ as defined in (8). Then for any unit vector $f \in L^{2}(\mathbf{R}, \mu)$,

$$
\left\langle U_{t}^{\mu} f, f\right\rangle=\int_{\mathbf{R}} e^{i t x}|f(x)|^{2} d \mu(x), \forall t \in \mathbf{R} .
$$

Since the probability measure $|f(x)|^{2} d \mu(x)$ is absolutely continuous w.r.t. $d \mu(x)$, it is clear that the spectral measure of $\left(U_{t}^{\mu}\right)$ is $\mu$. More generally, we have the following:

Proposition 4.3. Let $\mu$ be a symmetric probability measure on $\mathbf{R}$. Consider the unitary representation $\left(U_{t}^{\mu}\right)$ defined on $L^{2}(\mathbf{R}, \mu)$ by (8) . Then for any $n \geq 1$, the spectral measure of the $n$-fold tensor product $\left(U_{t}^{\mu}\right)^{\otimes n}$ is the $n$-fold convolution product

$$
\mu^{* n}=\underbrace{\mu * \cdots * \mu}_{n \text { times }}
$$

4.2. Examples of solid $\mathrm{II}_{1}$ factors. Erdös showed in [9] that the symmetric probability measure $\mu_{\theta}$, with $\theta=5 / 2$, obtained as the weak limit of

$$
\left(\frac{1}{2} \delta_{-\theta^{-1}}+\frac{1}{2} \delta_{\theta^{-1}}\right) * \cdots *\left(\frac{1}{2} \delta_{-\theta^{-n}}+\frac{1}{2} \delta_{\theta^{-n}}\right)
$$

has a Fourier Transform

$$
\widetilde{\mu}_{\theta}(t)=\prod_{n \geq 1} \cos \left(\frac{t}{\theta^{n}}\right)
$$

which vanishes at infinity, i.e. $\widetilde{\mu}(t) \rightarrow 0$, as $|t| \rightarrow \infty$, and $\mu_{\theta}$ is singular w.r.t. the Lebesgue measure $\lambda$.

Example 4.4. Modifying the measure $\mu_{\theta}$, Antoniou \& Shkarin (see Theorem 2.5, v in [2]) constructed an example of a symmetric probability $\mu$ on $\mathbf{R}$ such that:

(1) The Fourier Transform of $\mu$ vanishes at infinity, i.e. $\widetilde{\mu}(t) \rightarrow 0$, as $|t| \rightarrow \infty$.

(2) For any $n \geq 1$, the $n$-fold convolution product $\mu^{* n}$ is singular w.r.t. the Lebesgue measure $\lambda$.

Let $\mu$ be a symmetric probability measure on $\mathbf{R}$ as in Example 4.4. Proposition 4.1 and Proposition 4.3 yields that the unitary representation $\left(U_{t}^{\mu}\right)$ defined on $L^{2}(\mathbf{R}, \mu)$ by (8) satisfies:

(1) $\left(U_{t}^{\mu}\right)$ is mixing.

(2) The spectral measure of $\bigoplus_{n \geq 1}\left(U_{t}^{\mu}\right)^{\otimes n}$ is singular w.r.t. the Lebesgue measure $\lambda$.

Let now $\mathcal{M}=\Gamma\left(H_{\mathbf{R}}, U_{t}\right)^{\prime \prime}$ and let $M=\mathcal{M} \rtimes_{\sigma} \mathbf{R}$ be the continuous core. Let $q \in L(\mathbf{R})$ be a non-zero projection such that $\operatorname{Tr}(q)<\infty$. Denote by $N=q M q$ the corresponding $\mathrm{II}_{1}$ factor. Using free probability techniques such as the free entropy, Shlyakhtenko (see Theorem 9.12 in [30]) showed that if the spectral measure of the unitary representation $\bigoplus_{n>1} U_{t}^{\otimes n}$ is singular w.r.t. the Lebesgue measure $\lambda$, then for any finite set of generators $X_{1}, \ldots, X_{n}$ of $N$, the free entropy dimension satisfies

$$
\delta_{0}\left(X_{1}, \ldots, X_{n}\right) \leq 1 .
$$

In particular, $N$ is not isomorphic to any interpolated free group factor $L\left(\mathbf{F}_{t}\right)$, for $1<t \leq \infty$. Combining these two results together with Corollary 3.7, we obtain the following: 
Theorem 4.5. Let $\mu$ be a symmetric probability measure on $\mathbf{R}$ as in Example 4.4. Let $\mathcal{M}=\Gamma\left(K_{\mathbf{R}}^{\mu}, U_{t}^{\mu}\right)^{\prime \prime}$ be the free Araki-Woods factor associated with the orthogonal representation $\left(U_{t}^{\mu}\right)$ acting on the real Hilbert space $K_{\mathbf{R}}^{\mu}$, as defined in $(8-9)$. Let $M=\mathcal{M} \rtimes_{\sigma} \mathbf{R}$ be the continuous core. Fix a non-zero projection $q \in L(\mathbf{R})$ such that $\operatorname{Tr}(q)<\infty$, and denote by $N=q M q$ the corresponding $\mathrm{II}_{1}$ factor. Then

(1) $N$ is non-amenable and solid.

(2) $N$ has full fundamental group, i.e. $\mathcal{F}(N)=\mathbf{R}_{+}^{*}$.

(3) $N$ is not isomorphic to any interpolated free group factor $L\left(\mathbf{F}_{t}\right)$, for $1<t \leq \infty$.

We believe that all the free Araki-Woods factors $\mathcal{M}=\Gamma\left(H_{\mathbf{R}}, U_{t}\right)^{\prime \prime}$ have the complete metric approximation property (c.m.a.p.), i.e. there exists a sequence $\Phi_{n}: \mathcal{M} \rightarrow \mathcal{M}$ of finite rank, completely bounded maps such that $\Phi_{n} \rightarrow$ Id ultraweakly pointwise, as $n \rightarrow \infty$, and $\limsup _{n \rightarrow \infty}\left\|\Phi_{n}\right\|_{\mathrm{cb}} \leq 1$. If $\mathcal{M}=\Gamma\left(H_{\mathbf{R}}, U_{t}\right)^{\prime \prime}$ had the c.m.a.p. then by [1], the continuous core $M=\mathcal{M} \rtimes_{\sigma} \mathbf{R}$ would have the c.m.a.p., as well as the $\mathrm{II}_{1}$ factor $q M q$, for $q \in M$ non-zero finite projection. On the other hand, the wreath product $\mathrm{II}_{1}$ factors $L\left(\mathbf{Z} \imath \mathbf{F}_{n}\right)$ do not have the c.m.a.p., for any $2 \leq n \leq \infty$, by [18. Thus, we conjecture that the solid $\mathrm{II}_{1}$ factors constructed in Theorem 4.5 are not isomorphic to $L\left(\mathbf{Z} \imath \mathbf{F}_{n}\right)$, for any $2 \leq n \leq \infty$.

\section{REFERENCES}

[1] C. Anantharaman-Delaroche, Amenable correspondences and approximation properties for von Neumann algebras. Pacific J. Math. 171 (1995), 309-341.

[2] I. Antoniou \& S. A. ShKarin, Decay measures on locally compact abelian topological groups. Proc. Roy. Soc. Edinburgh 131 (2001), 1257-1273.

[3] L. BARnett, Free product von Neumann algebras of type III. Proc. Amer. Math. Soc. 123 (1995), $543-553$.

[4] I. Chifan \& A. IOANA, Ergodic subequivalence relations induced by a Bernoulli action. arXiv:0802.2353

[5] I. Chifan \& C. Houdayer, Bass Serre rigidity results in von Neumann algebras. Duke Math. J., to appear. arXiv:0805.1566

[6] A. Connes, Almost periodic states and factors of type $\mathrm{III}_{1}$. J. Funct. Anal. 16 (1974), 415-445.

[7] A. Connes, Une classification des facteurs de type III. Ann. Sci. École Norm. Sup. 6 (1973), 133-252.

[8] K. Dykema, Interpolated free group factors. Pacific J. Math. 163 (1994), 123-135.

[9] P. ERdös, On a family of symmetric Bernoulli convolutions. Amer. J. Math. 61 (1939), 974-976.

[10] U. HaAgerup, Connes' bicentralizer problem and uniqueness of the injective factor of type III $_{1}$. Acta Math. 69 (1986), 95-148.

[11] C. Houdayer, Free Araki-Woods factors and Connes' bicentralizer problem. Proc. Amer. Math. Soc. 137 (2009), 3749-3755.

[12] C. HoudAYER, Construction of type $\mathrm{II}_{1}$ factors with prescribed countable fundamental group. J. reine angew Math. 634 (2009), 169-207.

[13] C. Houdayer, On some free products of von Neumann algebras which are free Araki-Woods factors. Int. Math. Res. Notices. Vol. 2007, article ID rnm098, 21 pages.

[14] A. Ionna, J. Peterson \& S. Popa, Amalgamated free products of w-rigid factors and calculation of their symmetry groups. Acta Math. 200 (2008), 85-153.

[15] N. Ozawa, Solid von Neumann algebras. Acta Math. 192 (2004), 111-117.

[16] N. Ozawa A Kurosh-type theorem for type $\mathrm{II}_{1}$ factors. Int. Math. Res. Notices. Vol. 2006 : article ID 97560, 21 pages.

[17] N. OzawA, An example of a solid von Neumann algebra. Hokkaido Math. J., 38 (2009), 557-561.

[18] N. Ozawa \& S. PopA, On a class of $\mathrm{II}_{1}$ factors with at most one Cartan subalgebra. Ann. of Math., to appear arXiv:0706.3623

[19] S. PopA, On the superrigidity of malleable actions with spectral gap. J. Amer. Math. Soc. 21 (2008), 981-1000.

[20] S. PopA, On Ozawa's property for free group factors. Int. Math. Res. Notices. Vol. 2007 : article ID rnm036, 10 pages.

[21] S. PopA, Strong rigidity of $\mathrm{II}_{1}$ factors arising from malleable actions of w-rigid groups I. Invent. Math. 165 (2006), 369-408.

[22] S. POPA, On a class of type $\mathrm{II}_{1}$ factors with Betti numbers invariants. Ann. of Math. 163 (2006), 809-899. 
[23] S. PopA, Some rigidity results for non-commutative Bernoulli Shifts. J. Funct. Anal. 230 (2006), 273328.

[24] S. Popa \& S. VAes, Strong rigidity of generalized Bernoulli actions and computations of their symmetry groups. Adv. Math. 217 (2008), 833-872.

[25] F. RĂDUlESCU, Random matrices, amalgamated free products and subfactors of the von Neumann algebra of a free group, of noninteger index. Invent. Math. 115 (1994), 347-389.

[26] F. RĂDUlescu, A one-parameter group of automorphisms of $L\left(\mathbf{F}_{\infty}\right) \otimes \mathbf{B}(H)$ scaling the trace. C. R. Acad. Sci. Paris Sr. I Math. 314 (1992), 1027-1032.

[27] D. Shlyakhtenko, Some estimates for non-microstates free entropy dimension, with applications to q-semicircular families. Int. Math. Res. Notices 51 (2004), 2757-2772.

[28] D. Shlyakhtenko, On the classification of full factors of type III. Trans. Amer. Math. Soc. 356 (2004), 4143-4159.

[29] D. Shlyakhtenko, On multiplicity and free absorption for free Araki-Woods factors. math.0A/0302217

[30] D. Shlyakhtenko, A-valued semicircular systems. J. Funct. Anal. 166 (1999), 1-47.

[31] D. Shlyakhtenko, Some applications of freeness with amalgamation. J. reine angew. Math. 500 (1998), 191-212.

[32] D. Shlyakhtenko, Free quasi-free states. Pacific J. Math. 177 (1997), 329-368.

[33] M. TAKESAKI, Duality for crossed products and structure of von Neumann algebras of type III. Acta Math. 131 (1973), 249-310.

[34] Y. UedA, Amalgamated free products over Cartan subalgebra. Pacific J. Math. 191 (1999), 359-392.

[35] S. VAes \& R. Vergnioux, The boundary of universal discrete quantum groups, exactness and factoriality. Duke Math. J. 140 (2007), 35-84.

[36] S. VAes, Rigidity results for Bernoulli actions and their von Neumann algebras (after S. Popa). Séminaire Bourbaki, exposé 961. Astérisque 311 (2007), 237-294.

[37] S. VAEs, États quasi-libres libres et facteurs de type III (d'après D. Shlyakhtenko). Séminaire Bourbaki, exposé 937, Astérisque 299 (2005), 329-350.

[38] D.-V. Voiculescu, K.J. Dykema \& A. Nica, Free random variables. CRM Monograph Series 1. American Mathematical Society, Providence, RI, 1992.

CNRS-ENS Lyon, UMPA UMR 5669, 69364 Lyon CEDEx 7, France

E-mail address: cyril.houdayer@umpa.ens-lyon.fr 